\title{
Automatic classification of eclipsing binaries light curves using neural networks ${ }^{\star}$
}

\author{
L. M. Sarro ${ }^{1}$, C. Sánchez-Fernández ${ }^{2,3}$, and Á. Giménez ${ }^{4}$
}

\author{
1 Dpt. de Inteligencia Artificial, U.N.E.D., c/ Juan del Rosal, 16, 28040 Madrid, Spain \\ e-mail: 1sb@dia.uned.es \\ 2 Laboratorio de Astrofísica Espacial y Física Fundamental, PO Box 50727, 28080 Madrid, Spain \\ 3 XMM-Newton SOC, ESAC, PO Box 50727, 28080 Madrid, Spain \\ e-mail: Celia.Sanchez@sciops.esa.int \\ 4 Research and Scientific Support Department, ESA, ESTEC, Postbus 299, 2200 AG Noordwijk, The Netherlands \\ e-mail: agimenez@rssd.esa.int
}

Received 7 February 2005 / Accepted 26 August 2005

\section{ABSTRACT}

In this work we present a system for the automatic classification of the light curves of eclipsing binaries. This system is based on a classification scheme that aims to separate eclipsing binary systems according to their geometrical configuration in a modified version of the traditional classification scheme. The classification is performed by a Bayesian ensemble of neural networks trained with Hipparcos data of seven different categories including eccentric binary systems and two types of pulsating light curve morphologies.

Key words. stars: binaries: eclipsing - stars: binaries: general - methods: data analysis - methods: statistical

\section{Introduction}

Eclipsing binaries (hereafter EBs) play a fundamental role in modern astrophysics for several reasons. First of all, detached double-lined EBs without mass transfer between the components are a prime tool to derive fundamental stellar parameters; joint analysis of their light and radial velocity curves provides accurate $(1-2 \%)$ determinations of masses, radii, and luminosity ratios. Eclipsing binaries also work as testing grounds for stellar structure and evolution models, and as such they play a key astrophysical role across the whole HR diagram. Recently, the study of EBs in other galaxies and clusters has made it possible to explore stellar evolution and to establish mass-luminosity laws for galaxies with a vastly different evolutionary and chemical histories from our own Galaxy (such as LMC and SMC). Moreover, EBs are beginning to play an important role in cosmology as distance indicators to nearby galaxies. Studies of Galactic early-type binaries have shown that distance moduli accurate to $\pm 0.1 \mathrm{mag}$ are attainable, a precision comparable to that obtained for individual Cepheid variables. As more data are accumulated, studies of these systems may lead to an improvement in the extragalactic distance scale.

In recent years, large scale photometric surveys have been providing a wealth of light curves of variable stars out of which a large amount of EB systems can be selected. For example,

* Tables 4-7 and Sect. 3 as an appendix are only available in electronic form at http://www. edpsciences.org the ESA astrometric satellite Hipparcos found $70 \%$ new variables out of the relatively bright selected sample. The GAIA large-scale photometric survey will also have significant scientific value for the study of nearly all types of variable stars, including eclipsing binaries. It is expected that about 1 million EBs, those with $V \leq 16 \mathrm{mag}$, will be discovered. Even if reliable physical parameters could be derived for only $1 \%$ of the observed EBs, this would be a great contribution to stellar astrophysics in comparison with what has been obtained so far from ground-based observations. The Optical Monitoring Camera (OMC; Mas-Hesse et al. 2003) onboard INTEGRAL is another example of an instrument that continuously provides high quality photometric measurements of thousands of eclipsing and pulsating variables, amongst other objects more closely related to high energy astrophysics. Finally, the COnvection ROtation and planetary Transits (COROT) mission will produce, as a by product, enormous amounts of light curves of objects with unprecedented accuracy (see e.g. Baglin et al. 2002). All these vast amounts of data offer the opportunity to select not only EB light curves, but all kinds of light curves for deeper investigation and/or follow-up. Such databases also provide astronomers with powerful heuristics like the possibility to construct statistically significant samples of objects that can be used as probes for correlations between physical parameters, e.g., in the case of the rotation-activity correlation (Jordan \& Montesinos 1991). Nevertheless and despite all this encouraging prospects, it is becoming increasingly clear that 
intelligent processing of these large datasets is needed, and no method based on manual procedures can be used. It is precisely the enormity of this volume of data that makes it necessary to implement automatic light curve classification tools before any serious scientific analysis. Fortunately, it is exactly in these kinds of tasks (such as pattern recognition, classification, clustering, and knowledge discovery in the form of dimensional correlations) that machine learning and artificial intelligence techniques yield their best performances.

In this paper we concentrate on the applications of neural networks for the task of light curve identification and clustering. Neural networks have been widely used in the past for classification of stellar (Snider et al. 2001) and galactic (Folkes et al. 1996) spectra, star/galaxy separation in images (Philip et al. 2002; Cortiglioni et al. 2001), or quasar detection. Sometimes the lassification process takes input data spanning a combination of spatial and temporal dimensions as in the case of solar flare detection where time series of images are used for the classification of events (Fernandez Borda et al. 2002). They have also been used for time series prediction (Verdes et al. 2000), nonlinear system identification (Bailer-Jones 2000; Carroll \& Staude 2001), and telescope control (Sandler et al. 1991) to cite but a few. In the specific field of light curve analysis, neural networks have been used recently for clustering purposes (Brett et al. 2004) and for microlensing detection (Belokurov et al. 2003; Belokurov et al. 2004). Here we present a refined classifier for eclipsing binaries based on stateof-the-art neural networks that builds upon some of the work presented in these previous developments.

In this work we apply Bayesian techniques to the training of neural networks for the automatic classification of light curves of variable stars based solely on their morphological aspects. The network is able to recognize four types of eclipsing binary systems and two types of pulsating star light curves. Furthermore, all the types define a link between the morphology of the light curve and the underlying physical scenarios as much as possible. In Sect. 2 we describe the classification scheme in detail; in Sect. 3 we describe the preprocessing of the data and the neural network architecture and training; in Sect. 4 we describe the results obtained, assess the quality and performance of the system and analyse the resulting connection topology; finally, in Sect. 5, we summarise the conclusions of this work.

\section{Classification scheme}

One obvious requirement of any classification system is the direct link between the features used as input and the classes defined from them. In the realm of variable systems, unfortunately, we find that either the classes established up to now are not consistently defined in terms of the light curves, as in the case of eclipsing binaries, or there are degeneracies, as in the case of pulsating stars, in the sense that different categories can have morphologically identical light curves. With pulsating variable light curves, the degeneracy can only be resolved with supplementary spectral information and periods. This problem will be addressed in a future paper where a multi-agent expert system will be presented, which is capable of classifying pulsating stars (identified by their light curves) by navigating the Virtual Observatory space searching for discriminant observations. Here we restrict ourselves to the problem of separating pulsating variables from eclipsing binary systems and subclassifying the latter into physically inspired classes univocally defined in terms of their light curves.

The two main factors that determine the shape of the light curve of an eclipsing binary system are its geometric configuration (i.e. the size of the component stars relative to their Roche lobes), which determines the fraction of the light curve occupied by eclipses, and the relative brightness of the stellar components, which determines the eclipse depths. The inclination of the system with respect to the line of sight can affect the depths of the eclipses, but its effect on the overall light curve morphology is less important.

We propose here a classification scheme which aims to separate eclipsing binary systems according to their geometrical configuration. This scheme is adapted from the historical classification of eclipsing binary light curves into three groups (Algol, Beta Lyrae, and W Ursae Majoris), but attempts to solve the problems of class heterogeneity and subjectivity of the traditional light curve classification, which includes systems with different physical properties in the same group. Our classification relates the groups established to the geometry, in the sense that systems with the same geometrical configuration are classified in the same group.

We note here a previous attempt to solve the degeneracy of the traditional classification of eclipsing binaries light curves by Alcock et al. (1997). They proposed a decimal classification scheme based on combining the relative radii of the stars and the surface relative flux ratio. As an alternative, when only the light curve morphology is available, we found that a simple 4-group classification scheme suffices to separate the systems into homogeneous classes.

\subsection{Our classification}

Definition of the classes assumes that the light curve has been processed such that the phase of the deeper eclipse (we refer to primary eclipse) is defined as 0.75 . Systems are classified into 4 groups as follows:

- Class 1 systems: light curves with well-defined start and end to both eclipses. These light curves may present small curvatures out of eclipses, but this curvature never masks the beginning and end of the occultations.

- Class 2 systems: light curves with only a well-defined primary eclipse, while the secondary has no clear beginning or end.

- Class 3 systems: light curves with eclipses of different depth and no flat light curves out of ecplise. In these systems, the light curve curvature out of eclipse masks the beginning and end of the occultations.

- Class 4 systems: light curves with the equal depth eclipses alternating, and no flat light curve out of eclipse.

Figure 1 shows example light curves from the Hipparcos catalogue for each class. 

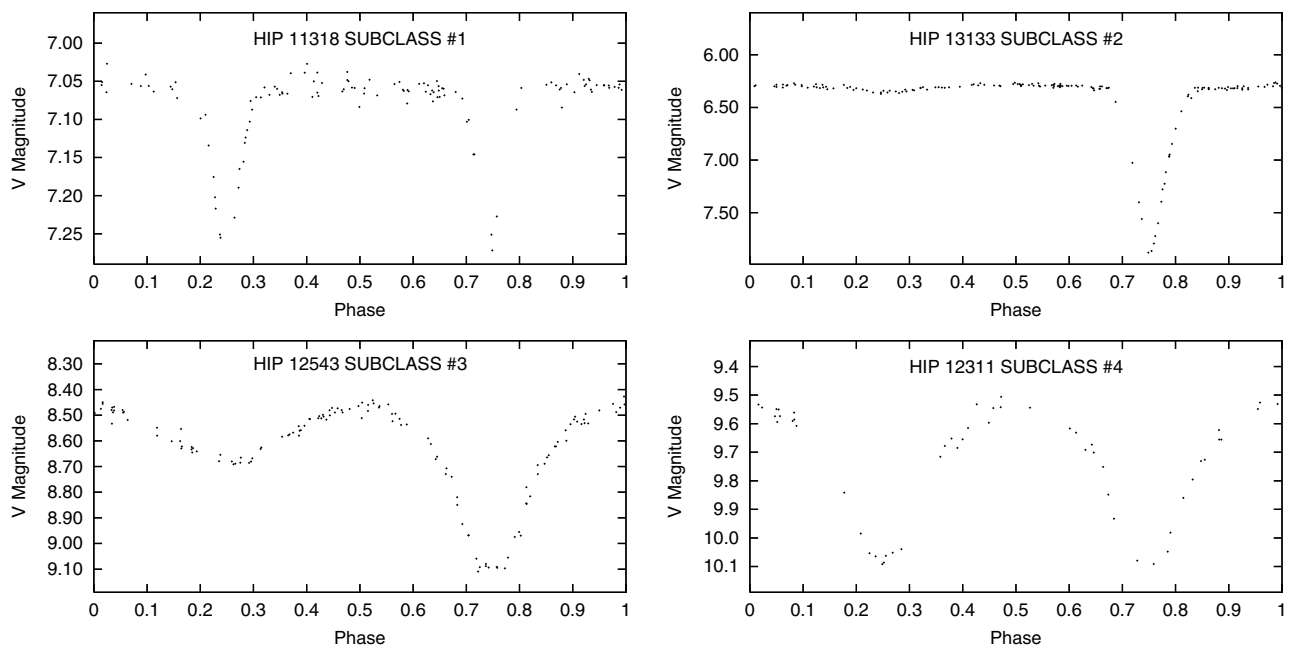

Fig. 1. Examples of the classes defined in the text obtained by Hipparcos, folded with the periods provided in the mission catalogue.

Table 1. Number of light curves of each class used in the training set. Type 0 includes eccentric systems from the Hipparcos catalogue plus 112 synthetic light curves (see text).

\begin{tabular}{ccccccc}
\hline \hline Type 0 & Type 1 & Type 2 & Type 3 & Type 4 & Type A & Type B \\
\hline $32(+112)$ & 269 & 164 & 192 & 129 & 131 & 693 \\
\hline
\end{tabular}

Table 2. Number of light curves of each class of pulsation used in the training set (pulsation class taken from the Hipparcos catalogue).

\begin{tabular}{ccccccccc}
\hline \hline & $\alpha$ Cygni & $\beta$ Cephei & Cepheids & W Virginis & $\delta$ Cepheids & $\delta$ Scuti & Mira & RR Lyrae \\
\hline Type A & 5 & 26 & 2 & 2 & 20 & 51 & 19 & 6 \\
Type B & 11 & 20 & 17 & 23 & 226 & 36 & 182 & 178 \\
\hline
\end{tabular}

\subsection{Application to a sample of systems}

In order to show the relation between the classes established by this scheme and some of the system parameters, we classified a set of 81 binary systems with well-studied light curves and precisely determined physical parameters. The list of systems used in this study and their main physical parameters can be found in Tables 4-7.

In the following, we will analyse the classes in terms of the component masses, orbital separation, mass ratio, and filling factors of the 81 systems included in our sample. In order to help with the interpretation of the combination of any two such parameters, we first show in Fig. 2 the sample masses, orbital separations and, mass ratio for the systems classified in each class. We can see in the total mass plot that there is no discriminant boundary or general trend between classes, although type 4 systems seem to be characterized by a lower mass. Orbital separations, on the other hand, show a decreasing trend towards higher types. We see how an apparent segregation in the total masses of type 3 systems into two sets (low and high mass objects) is reproduced in the separation plot in the sense that the less massive systems also have lower orbital separations and vice versa. This segregation into two groups may be an artifact caused by a limited sample size. Thus, more systems of this class with accurately determined parameters are needed to clarify whether two different populations with similar light curves indeed exist or whether there is continuous transition. Finally, the mean value of the mass ratio of the components shows values closer to 1 for type 1 systems, lowest values for type 2, and increasing values thereafter (types 3 and 4).

If we now plot the radius to orbital separation ratio for both components of each system as a function of the mass ratio $q$ (defined as $q=M_{2} / M_{1}, M_{1}$ being the most massive star), we obtain Figs. 3 and 4 where we have also included the Roche lobe size (in orbital separation units) computed using the approximation of Eggleton (1983). Figure 3 clearly shows that primary components of type 1 and 2 systems are well below the Roche lobe radius, while type 3 primaries are close to it, and type 4 primary stars clearly fill their Roche lobes. At the same time, only radii of type 1 system secondary stars are clearly below the Roche limit. Under this perspective, it is evident that our classification scheme is a morphological transposition of the different geometrical configurations: type 1 systems are composed of two stars with radii clearly below the Roche lobe (detached systems); type 2 systems are composed of a primary star well below its Roche limit and a secondary filling its Roche lobe (i.e. semidetached systems); type 3 systems have a primary component close to filling its Roche lobe and a secondary component already filling the critical lobe and therefore, they represent semidetached systems close to contact; finally, type 4 light curves represent contact binaries with both components filling their Roche lobes and possibly exceeding them. We will pursue further the implications of this scheme after considering 
Table 3. Cross-class misclassification percentages. Each row lists the percentage of light curves of a given class that were mistakenly classified as belonging to the corresponding type in the row of headers.

\begin{tabular}{cccccccc}
\hline \hline & Type 0 & Type 1 & Type 2 & Type 3 & Type 4 & Type A & Type B \\
\hline Type 0 & - & $3.6 \pm 0.6$ & $0.5 \pm 0.3$ & $0.3 \pm 0.3$ & $0.0 \pm 0.0$ & $0.0 \pm 0.0$ & $0.0 \pm 0.0$ \\
Type 1 & $1.0 \pm 0.3$ & - & $1.5 \pm 0.4$ & $4.9 \pm 0.7$ & $1.6 \pm 0.3$ & $0.0 \pm 0.0$ & $0.0 \pm 0.0$ \\
Type 2 & $0.2 \pm 0.2$ & $4.0 \pm 0.9$ & - & $0.0 \pm 0.0$ & $0.0 \pm 0.0$ & $0.0 \pm 0.0$ & $0.0 \pm 0.0$ \\
Type 3 & $0.0 \pm 0.0$ & $8.8 \pm 1.7$ & $0.0 \pm 0.0$ & - & $5.3 \pm 1.1$ & $0.0 \pm 0.0$ & $0.0 \pm 0.0$ \\
Type 4 & $0.0 \pm 0.0$ & $2.1 \pm 0.7$ & $0.0 \pm 0.0$ & $7.4 \pm 1.0$ & - & $0.0 \pm 0.0$ & $0.0 \pm 0.0$ \\
Type A & $0.0 \pm 0.0$ & $0.0 \pm 0.0$ & $0.0 \pm 0.0$ & $0.0 \pm 0.0$ & $0.0 \pm 0.0$ & - & $13.4 \pm 1.7$ \\
Type B & $0.0 \pm 0.0$ & $0.2 \pm 0.1$ & $0.0 \pm 0.0$ & $0.0 \pm 0.0$ & $0.0 \pm 0.0$ & $3.2 \pm 0.4$ & - \\
\hline
\end{tabular}

possible correlations between total mass, orbital separation and, mass ratios.

Figure 5 represents all systems in the sample in the $\log \left(M_{\text {tot }}\right)-\log (a)$ space, with $M_{\text {tot }}$ the system total mass and $a$ the orbital separation in solar radius units. Although there is clearly no separability in this space, there are evident trends in the data. Again, type 4 systems are found in the low orbital separation and low total mass region of the plot, and seem to follow a tight linear relation. The rest of the types continue this correlation with increasing values of the dispersion: low mass type 3 systems follow the trend to the right with higher values of both parameters, then type 2 systems, and finally, with a high degree of overlapping, type 1 systems occupy the high total mass, high orbital separation region of the plot.

Once revised, the physical characteristics of the proposed classes, we can reformulate the definitions, this time summarizing the regions of parameter space where we can expect to find the system.

\subsubsection{Systems with type 1 light curves}

These are detached systems with widely varying total masses and a wide range of spectral types from $\mathrm{O}$ to $\mathrm{F}$. Most of the systems assigned to this class have mass ratios close to 1 due to selection effects. Both components are well within the Roche lobe and have orbital separations in the $10-100 R_{\odot}$ range. All these properties result in light curves with well-defined beginnings and ends of both eclipses and flat regions outside them. These binaries are the best source of information to study stellar absolute dimensions and structure. Most systems included in this group are eccentric.

\subsubsection{Systems with type 2 light curves}

Systems classified as type 2 have low mass ratios and total masses below $10 M_{\odot}$. In the systems studied, the primary component of spectral type A or B is in the hydrogen burning phase, and the secondary component (that originally transferred a significant fraction of its mass through the $L_{1}$ Lagrange point ) has spectral type $\mathrm{G}$ or $\mathrm{K}$ and has a mass around or below the solar mass. These systems have their origin in detached systems in which the most massive star evolved out of the main sequence, filled its Roche lobe, and transferred mass to the secondary component through $L_{1}$ until the original secondary
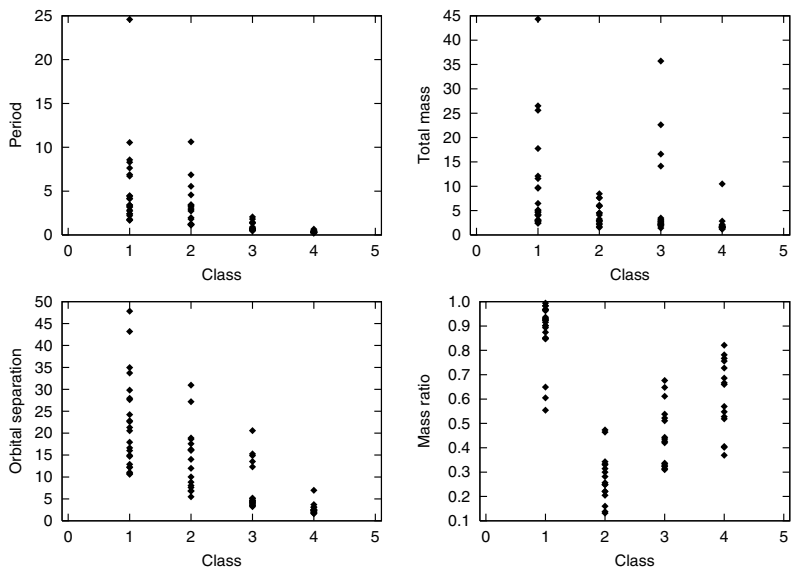

Fig. 2. System parameters grouped by class as defined in the text. Orbital periods (days) and separations (expressed in solar radii) are shown in the left column of the plot; total mass of the system (expressed in solar masses) and mass ratios are shown in the right column plots.

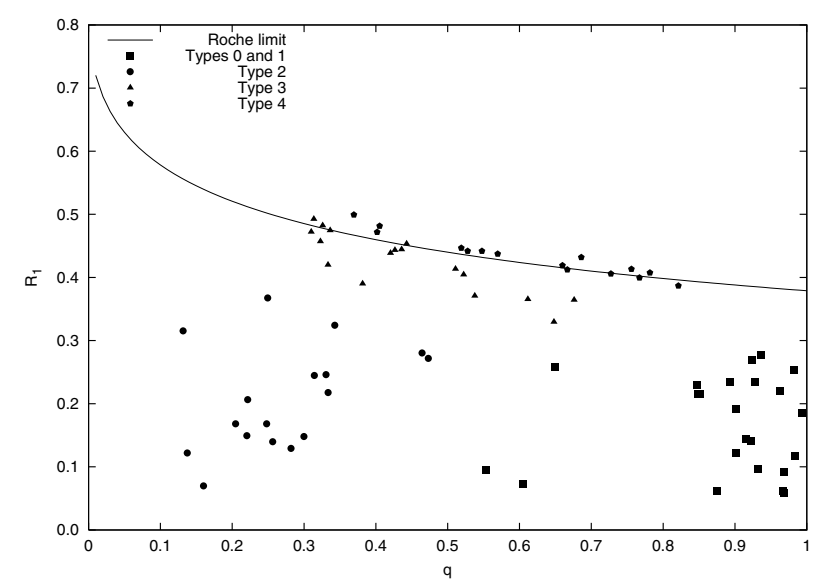

Fig. 3. Radius to orbital separation ratio of the primary components in the sample as a function of the mass ratio $q$.

became the most massive component. Although the details of the process remain unclear (Hall 1975; Ziolkowski 1976), system mass loss cannot be discarded. 


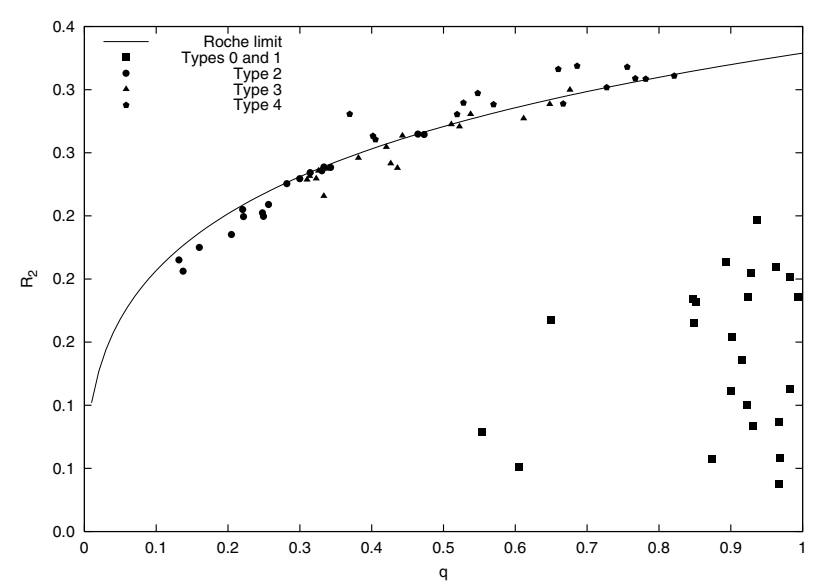

Fig. 4. Radius to orbital separation ratio of the secondary components in the sample as a function of the mass ratio $q$.

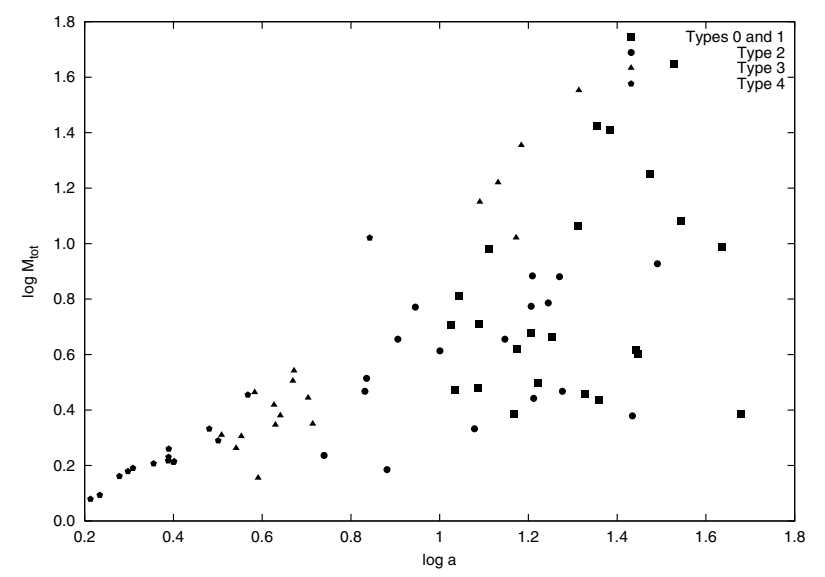

Fig. 5. Logarithmic representation of the eclipsing binary system total mass $\left(M_{\mathrm{tot}}\right)$ as a funtion of the orbital separation $a$ in solar radius units.

\subsubsection{Systems with type 3 light curves}

Type 3 systems in the sample show two different clusters in parameter space. They are all semidetached systems with the primary component close to filling the Roche lobe and, due to the proximity of the components, both eclipses alternate without intereclipse flat intervals.

The most populated cluster is composed of systems with total masses lower than $5 M_{\odot}$ and is characterized by short period (less than 1 day) orbits and small orbital separations in close-to-contact configurations. The primary components are spectral types A or F, and the secondary stars are one or two types cooler.

The second, less populated cluster of systems corresponds to total masses above $10 M_{\odot}$ and periods in the 1-3 days range. They show moderate mass ratios, and both components are of similar spectral types around B. Again, the more massive primary component is close to its Roche lobe but separated from it, and the evolved secondary is in contact with its lobe. They probably originated in very close orbits, with mass ratios around 1 , and evolved to near contact configurations as the stars expanded as a consequence of Main Sequence evolution.

\subsubsection{Systems with type 4 light curves}

All light curves classified as type 4 correspond to systems in contact where both stars fill and possibly exceed their Roche lobes. If they exceed this limit, the size of the common envelope depends on the most external contact surface. These systems are characterized by short periods, small orbital separations, and a wide range of mass ratios. Except for RZ Pyx with a spectral type $\mathrm{B}$, the rest of the systems are composed of late type stars with total masses below $3 M_{\odot}$. It is not yet clear whether these systems are formed as contact binaries or if they evolve from detached systems through loss of angular momentum. Most possibly, the population of contact binaries is a mixture of both evolutionary paths.

To finish this section we would like to point out that, unfortunately, our scheme is not without degeneracies or crossclass contamination. The main sources of contamination arise from pre-main sequence detached systems. In these systems, one of the components is in the contracting phase towards the Main Sequence while the second component has already stabilized in it. The former is far dimmer than the latter and can thus be a source of confusion with type 2 systems despite their detached geometry. Due to the relative youth of these pre-main sequence systems they generally have not had enough time to circularise their orbits and show therefore some degree of eccentricity. This criteria can be used to place them correctly in the type 1 group. Nevertheless, certain orientations of the orbit with respect to the observer may result in eclipses being in quadrature despite the eccentricity of the system.

\section{Results and discussion}

In order to assess the performance of the ensemble of neural networks thus generated, we divided the whole set of examples into two groups: (i) a training set used to obtain the a posteriori probabilities of the parameter sets generated by MCMC methods (75\% of the complete set), and (ii) a test set used to obtain estimates of the expected cross-class misclassification rates ( $25 \%$ of the complete set). In order to approximately maintain the relative size of each class in the complete set, a light curve is assigned to the training set with a 0.75 probability or to the test set with a 0.25 probability. This splitting is performed 10 times and the resulting blocks considered separately. Errors in the performance estimates correspond to the root sum square of the performance of the ten partitions. Furthermore, three different network architectures are tested. Invariably, all three architectures have a 50-unit input layer and a 7-unit output layer. They differ on the presence/absence of one or several hidden layers. The first network is a logistic regression network (with no hidden layer); the second network has one hidden layer with 30 units; and the third network architecture contains two hidden layers of 20 and 10 units, respectively. Each splitting of the complete set is used to generate 1000 networks of each architecture, and the last 200 are used to predict classes for the light curves in the corresponding test sets. Thus, we end up with $10 \times 3$ ensembles of 200 neural networks. In addition to this, the effectiveness of the ARD procedure was assesed by comparing the predictions on the test sets of networks of the 
same architecture with/without ARD implemented in the training process.

In order to avoid unnecessary computations, we checked the average error percentage for each architecture and found an $8.7 \% \pm 3.6$ for the $50-7$ architecture, $6.9 \% \pm 1.3$ for $50-30-7$ and $6.9 \% \pm 1.3$ for $50-20-10-7$. The average log probability of the test cases was -0.24 for the 50-7 network, -0.17 for the 50-30-7 network, and -0.18 for the 50-20-10-7 network. Although ARD could naturally prune unnecessary units and connections, if hyperparameters were introduced in all network layers, we preferred to continue the analysis with the 50-30-7 architecture.

The performance of the neural network does not depend on the total number of measurements in the light curve. It would indeed depend on the total information content of the available points (note that the total information content combines information not only on the phase coverage but also on the relevance of the covered phases for classification purposes), if no pattern completion were carried out during the preprocessing stage. This can be seen by taking the extreme case of an infinite number of points concentrated on a very narrow phase interval where all classes present the same behaviour. But, as explained above, the preprocessing stage completes the missing bins using the curvature of the closest light curves in the SOM. Therefore, if the completion process is correct and the initial incomplete light curve has enough information to reconstruct the missing phases, no dependence of the neural networks performance on the information content of the light curve before preprocessing should be detected, which is in fact the case down to the minimum information content found on Hipparcos light curves; around 20\%, 100\% is a complete light curve. Unfortunately, this robustness is not realistic since only a $10 \%$ of the catalogue has information content below $60 \%$, and therefore the statistics are rather poor. The study was carried out grouping the light curves in bins of information content width $20 \%$. The smaller number of cases in each bin increases the standard deviation up to $4.3 \%$.

We also investigated the dependence of the classifier performance on the ratio between the amplitude and the errors in the measurements of the light curve (the signal-to-noise ratio) and found no significant trend above a mean variance of $5.7 \%$. Again, the preprocessing stage tries to minimize the effect of the errors in the measurement by means of the regression process. It has to be beared in mind that it is actually a smooth curve (the result of the second regression) that is used as input to the neural net.

The robust performance of the neural network described in the preceding paragraphs can also be expected for light curves in the same information content and signal-to-noise ratio ranges as those found in the Hipparcos catalogue. As mentioned above, this implies light curves with information contents above $60 \%$ (although the classifier shows the same performance down to a $20 \%$ with only a few tens of light curves to compute the means). Light curves with lower information contents can possibly be mistankenly completed if not enough information is available for a reliable completion. Regarding the signal-to-noise ratios, we have found that $98.5 \%$ of the light curves in the catalogue have ratios above $5 \sigma$.

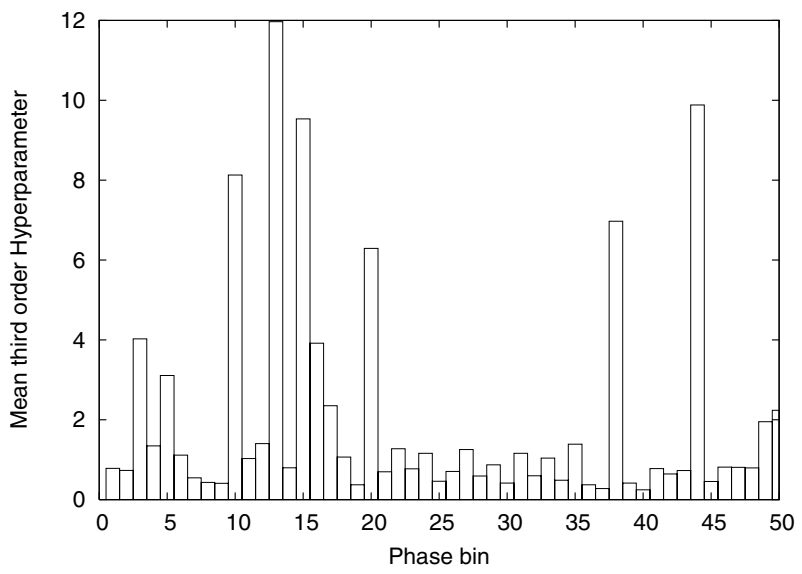

Fig. 8. Mean values of the hyperparameter controlling the average magnitude of the weights out of the input unit for the Hipparcos set plus 112 synthetic light curves of eccentric systems.

Finally, we studied the performance of the neural network as a function of the number of bins used as input and found that 50 bins lies in a plateau with similar performances that goes from 40 bins up to 90 bins. Below 40 bins the degradation is first due to the misclassification of eccentric systems and below 20 bins mainly to confusion between types 1 and 3 . Above 90 bins, there are not enough examples to construct the relationship between each input node and the class and the performance curve begins a slow decline as expected.

Table 3 shows the average cross-class misclassification percentage and the standard deviation computed for the 10 different splittings of the complete set for the 50-30-10 architecture. Each row lists the percentage of objects of a given type that have been misclassified in all other possible categories.

These percentages are less than 1 point lower on average than obtained without ARD implemented during the training. From this point onward, and having an estimate of the expected misclassification rate, we continue the analysis of the performance of our classification system with the complete set of Hipparcos plus synthetic eccentric light curves as training set of a 50-30-7 architecture network with ARD implemented. Although this particular choice is only marginally justified in terms of classification performance, we consider that it provides best results with maximum information. Figure 8 shows the mean values of the third level hyperparameter controlling the average strength of the connections out of each input unit, obtained in the last 1500 networks with ARD implemented.

It shows non negligible values at all phase bins although the average strength of neural connections from input units is seen to display some degree of structure. On a background of low magnitude weights, we find a higher concentration of sensitive units around phase 0.25 . This can be easily understood in terms of the classification criteria exposed in Sect. 2 and the preprocessing of the light curves, the combination of which makes the class assignment decision depend mainly on the properties of that region. The relative importance of the connection strength of synapsis out of the unit representing phase $\phi=0.75$ can be explained under the ARD framework as the result of the MCMC methods blindly exploring the hyperparameter space 
with a probability given only by the prior. This unit conveys no information at all (the preprocessing stage fixes its input at 1.0) and therefore, we can expect its posterior probability to be roughly equal for all possible values of this hyperparameter. The final value is simply an average of this blind exploration of the prior. The low sensitivity of input units away from $\phi=0.25$ can be explained by the easy separability of very eccentric systems with respect to all other classes.

\section{Conclusions}

In this work we present an automatic light curve classifier based on neural networks able to separate pulsating stars from eclipsing binary systems. We classified the latter into 4 groups, according to a new classification scheme based solely on the morphological features of the light curve, which maps the system geometrical configuration. We applied the new classification scheme to a sample of 81 systems with well-measured light curves and well-determined physical parameters, to investigate the physical properties of the classes thus defined. We found that, based only on the light curve morphology, we are able to separate systems with different geometrical configurations.

From a technical point of view, the improvement of our classification scheme with respect to the traditional one relies mainly establishing well-defined and objective criteria that can be easily implemented on a neural network. The traditional classification was not systematically formulated and was subjectively applied after visual inspection of the light curve by the observer. From a physical point of view, our classifcation scheme improves the traditional classification by establishing classes characterized by the variation of the system geometry from one group to the other. In the traditional one, systems with different geometrical configurations were classified in the same group.

We also considered under what circumstances the classification scheme proposed here would fail a priori to reflect the underlying geometrical configuration and found the following two exceptions:

- Pre-main-sequence systems with low luminosity secondaries and semimajor axis aligned with the visual line.

- Semi-detached systems with a secondary component in contact with the Roche lobe and a primary close to contact, being both stars of the same or similar luminosities.

We explored the classifier performance when trained with Hipparcos examples alone and together with a set of light curves artificially generated to increase the relative frequency of eccentric systems in the training set. In the latter case we found a significant improvement in the classifier's ability to detect eccentric binary systems at the expense of a small degradation in the overall performance. We explored several architectures for the network and found improved performance for networks with one or more hidden layers (with negligible differences between them). Finally, we also found negligible differences between the performance of classifiers trained with and without ARD. Almost the entire set of misclassifications occur at the boundaries between classes mainly due to the nonseparability of the sets of examples. We atribute this to the presence of noise in the training set. Nevertheless the softmax formulation of the model provides a quantitative measurement of the confidence in the class assignment in such cases, because systems in the proximity of a boundary between two classes exhibit comparable values in the output of the neurons that label those classes.

We have compared the performance of Bayesian neural networks presented above with that of a simple multilayer perceptron and found an overall improvement of $12.1 \%$; i.e., the percentage of right classifications of a 50-30-7 multilayer perceptron is $19.0 \%$. These figures combine both the improvement in the regression stage and that in the final classification. The inclusion of wide priors for the hyperparameters leads to increased robustness when outliers are expected. In our case, we have experienced that the results of regression with simple multilayer perceptrons, in the presence of outliers to the light curve, are significantly worse than those of Bayesian neural networks.

\section{References}

Alcock, C., Allsman, R. A., Alves, D., et al. 1997, AJ, 114, 326

Andersen, J. 1983, A\&A, 118, 255

Andersen, J. 1991, A\&ARv, 3, 91

Andersen, J., \& Clausen, J. V. 1989, A\&A, 213, 183

Andersen, J., Clausen, J. V., Giménez, A., \& Nordstroem, B. 1983a, A\&A, 128, 17

Andersen, J., Clausen, J. V., Nordstroem, B., \& Reipurth, B. 1983b, A\&A, 121, 271

Andersen, J., Clausen, J. V., Nordstrom, B., \& Popper, D. M. 1985, A\&A, 151, 329

Andersen, J., Clausen, J. V., \& Nordstrom, B. 1987, A\&A, 175, 60

Andersen, J., Clausen, J. V., Nordstrom, B., Gustafsson, B., \& Vandenberg, D. A. 1988, A\&A, 196, 128

Andersen, J., Clausen, J. V., \& Magain, P. 1989, A\&A, 211, 346

Andersen, J., Nordstrom, B., \& Clausen, J. V. 1990, A\&A, 228, 365

Baglin, A., Auvergne, M., Barge, P., et al. 2002, in Stellar Structure and Habitable Planet Finding, ESA SP-485, 17

Bailer-Jones, C. A. L. 2000, A\&A, 357, 197

Barone, F., Di Fiore, L., Milano, L., Pirozzi, L., \& Russo, G. 1992, Ap\&SS, 198, 321

Barone, F., Di Fiore, L., Milano, L., \& Russo, G. 1993, ApJ, 407, 237

Bell, S. A., \& Malcolm, G. J. 1987, MNRAS, 227, 481

Bell, S. A., Adamson, A. J., \& Hilditch, R. W. 1987a, MNRAS, 224, 649

Bell, S. A., Kilkenny, D., \& Malcolm, G. J. 1987b, MNRAS, 226, 879

Belokurov, V., Evans, N. W., \& Du, Y. L. 2003, MNRAS, 341, 1373

Belokurov, V., Evans, N. W., \& Le Du, Y. 2004, MNRAS, 352, 233

Bishop, C. M. 1995, Neural Networks for Pattern Recognition (Oxford, UK: Oxford University Press)

Bradstreet, D. H. 1985, ApJS, 58, 413

Breinhorst, R. A., Kallrath, J., \& Kaemper, B.-C. 1989, MNRAS, 241, 559

Brett, D. R., West, R. G., \& Wheatley, P. J. 2004, MNRAS, 353, 369

Carroll, T. A., \& Staude, J. 2001, A\&A, 378, 316

Cerruti-Sola, M., Milano, L., \& Scaltriti, F. 1981, A\&A, 101, 273

Cester, B., Fedel, B., Giuricin, G., Mardirossian, F., \& Pucillo, M. 1977, A\&A, 61, 469

Claret, A., Giménez, A., \& Martín, E. L. 1995, A\&A, 302, 741

Clausen, J. V. 1991, A\&A, 246, 397 
Clausen, J. V. 1996, A\&A, 308, 151

Clausen, J. V., \& Gronbech, B. 1976, A\&A, 48, 49

Clausen, J. V., \& Gronbech, B. 1977, A\&A, 58, 131

Clausen, J. V., \& Giménez, A. 1991, A\&A, 241, 98

Clausen, J. V., Gyldenkerne, K., \& Gronbech, B. 1976, A\&A, 46, 205

Clausen, J. V., Gyldenkerne, K., \& Gronbech, B. 1977, A\&A, 58, 121

Clausen, J. V., Giménez, A., \& Scarfe, C. 1986, A\&A, 167, 287

Cortiglioni, F., Mähönen, P., Hakala, P., \& Frantti, T. 2001, ApJ, 556, 937

Corvino, E., Barone, F., Milano, L., Russo, G., \& Sarna, M. J. 1990, MNRAS, 246, 472

De Greve, J. P. 1989, A\&A, 213, 195

Derman, E., \& Demircan, O. 1992, Ap\&SS, 189, 309

Drechsel, H., Haas, S., Lorenz, R., \& Mayer, P. 1994, A\&A, 284, 853

Eggleton, P. P. 1983, ApJ, 268, 368

Fernandez Borda, R. A., Mininni, P. D., Mandrini, C. H., et al. 2002, Sol. Phys., 206, 347

Folkes, S. R., Lahav, O., \& Maddox, S. J. 1996, MNRAS, 283, 651

Fracastoro, M. G. 1972, in An atlas of light curves of eclipsing binaries, Torino: Osservatorio Astronomico

Giménez, A., \& Clausen, J. V. 1986, A\&A, 161, 275

Giuricin, G., Mardirossian, F., \& Mezzetti, M. 1983, ApJS, 52, 35

Gronbech, B., Gyldenkerne, K., \& Jorgensen, H. E. 1977, A\&A, 55, 401

Gulmen, O., Sezer, C., \& Gudur, N. 1985, A\&AS, 60, 389

Hall, D. S. 1975, Acta Astron., 25, 215

Helt, B. E. 1987, A\&A, 172, 155

Hilditch, R. W. 1984, MNRAS, 211, 943

Hilditch, R. W., \& King, D. J. 1988, MNRAS, 231, 397

Hilditch, R. W., King, D. J., Hill, G., \& Poeckert, R. 1984, MNRAS, 208, 135

Hilditch, R. W., Skillen, I., Carr, D. M., \& Aikman, G. C. L. 1986, MNRAS, 222, 167

Hilditch, R. W., King, D. J., \& McFarlane, T. M. 1988, MNRAS, 231, 341

Hilditch, R. W., Hill, G., \& Khalesseh, B. 1992, MNRAS, 254, 82

Hrivnak, B. J. 1989, ApJ, 340, 458

Jeffreys, K. W. 1980, A\&AS, 42, 285

Jordan, C., \& Montesinos, B. 1991, MNRAS, 252, 21

Jorgensen, H. E., \& Gyldenkerne, K. 1975, A\&A, 44, 343

Karimie, M. T., \& Duerbeck, H. W. 1985, Ap\&SS, 117, 375

Khalesseh, B., \& Hill, G. 1992, A\&A, 257, 199

Khaliullin, K. F., \& Kozyreva, V. S. 1989, Ap\&SS, 155, 53

Khan, M. A., \& Budding, E. 1986, Ap\&SS, 125, 219

Kim, H.-I. 1989, ApJ, 342, 1061

Knipe, G. F. G. 1974, MNRAS, 167, 369

Kreiner, J. M., Pajdosz, G., Tremko, J., \& Zola, S. 1994, A\&A, 285, 459

Kwee, K. K., \& Van Genderen, A. M. 1983, A\&A, 126, 94

Lacy, C. H., \& Frueh, M. L. 1987, AJ, 94, 712

Lapasset, E., \& Claria, J. J. 1986, A\&A, 161, 264

Lapasset, E., \& Gomez, M. 1990, A\&A, 231, 365

Lapasset, E., Gomez, M., \& Farinas, R. 1996, PASP, 108, 332
Leung, K. C., \& Schneider, D. P. 1978, ApJ, 222, 917

Lipari, S. L., \& Sistero, R. F. 1986, MNRAS, 220, 883

Maceroni, C. 1986, A\&A, 170, 43

Maceroni, C., \& Van 'T Veer, F. 1996, A\&A, 311, 523

Maceroni, C., Vilhu, O., Van 'T Veer, F., \& Van Hamme, W. 1994, A\&A, 288, 529

Marton, S. F., Grieco, A., Lapasset, E., Sistero, R. F., \& Claria, J. J. 1990, A\&A, 237, 73

Martín, E. L., \& Rebolo, R. 1993, A\&A, 274, 274

Mas-Hesse, J. M., Giménez, A., Culhane, J. L., et al. 2003, A\&A, 411, L261

Maxted, P. F. L., \& Hilditch, R. W. 1995, A\&A, 301, 149

Maxted, P. F. L., \& Hilditch, R. W. 1996, A\&A, 311, 567

Maxted, P. F. L., Hill, G., \& Hilditch, R. W. 1994, A\&A, 282, 821

Maxted, P. F. L., Hill, G., \& Hilditch, R. W. 1995, A\&A, 301, 135

McFarlane, T. M., Bell, S. A., Adamson, A. J., \& Hilditch, R. W. 1986a, MNRAS, 223, 607

McFarlane, T. M., Hilditch, R. W., \& King, D. J. 1986b, MNRAS, 218, 159

Milano, L., Barone, F., Mancuso, S., Russo, G., \& Vittone, A. A. 1989, A\&A, 210, 181

Mochnacki, S. W. 1981, ApJ, 245, 650

Morris, S. L., \& Naftilan, S. A. 1997, AJ, 114, 2145

Neal, R. M. 1996, Bayesian Learning for Neural Networks (New York: Lecture Notes in Statistics Springer Verlag)

Olson, E. C., \& Plavec, M. J. 1997, AJ, 113, 425

Philip, N. S., Wadadekar, Y., Kembhavi, A., \& Joseph, K. B. 2002, A\&A, 385, 1119

Popper, D. M. 1980, ARA\&A, 18, 115

Popper, D. M. 1982, ApJ, 254, 203

Popper, D. M. 1987, ApJ, 313, L81

Richards, M. T., Mochnacki, S. W., \& Bolton, C. T. 1988, AJ, 96, 326

Rumelhart, D., Hinton, G., \& Williams, R. 1986, Nature, 323, 533

Samec, R. G. 1987, PASP, 99, 1310

Samec, R. G., \& Terrell, D. 1995, PASP, 107, 427

Samec, R. G., Gray, J. D., \& Carrigan, B. 1995, BAAS, 186, 4707

Sammon, J. W. 1969, IEEE Trans. Computers, 18, 401

Sandler, D. G., Barrett, T. K., Palmer, D. A., Fugate, R. Q., \& Wild, W. J. 1991, Nature, 351, 300

Sarma, M. B. K., Rao, P. V., \& Abhyankar, K. D. 1996, ApJ, 458, 371

Schneider, D. P., Darland, J. J., \& Leung, K. C. 1979, AJ, 84, 236

Snider, S., Allende Prieto, C., von Hippel, T., et al. 2001, ApJ, 562, 528

Van Gent, R. H. 1989, A\&AS, 77, 471

Van Hamme, W., \& Wilson, R. E. 1986, AJ, 92, 1168

Verdes, P. F., Parodi, M. A., Granitto, P. M., et al. 2000, Sol. Phys., 191, 419

Wachmann, A. A., Popper, D. M., \& Clausen, J. V. 1986, A\&A, 162, 62

Wilson, R. E., \& Devinney, E. J. 1973, ApJ, 182, 539

Wilson, R. E., \& Mukherjee, J. 1988, AJ, 96, 747

Zhai, D.-S., Zhang, X.-Y., \& Lu, W.-X. 1988, Ap\&SS, 146, 1

Ziolkowski, J. 1976, ApJ, 204, 512 


\section{Online Material}




\section{Neural classifier}

As mentioned in the introduction, the final aim of this work is to make the computational classification of automatically preprocessed light curves possible without human supervision. The classification system defined in the previous section was designed to accomplish this goal, while at the same time preserving the physical significance investigated there. In this section, we describe the methodology used to implement the classifier and the results obtained as assesed using standard techniques in the field of connectionism.

\subsection{Bayesian training of neural networks}

Most connectionist methods consist of distributing the computation of the solution of a given task amongst a number of interconnected, formally equivalent units or neurons performing very simple nonlinear operations upon the weighted sum of their inputs. The connection topology divides the ensemble of neurons (the neural network) in layers with forward connectivity. This architecture is commonly known as a multilayer perceptron. Although there are several other architectures and different local operations from the one sketched above, the multilayer perceptron is by far used the most for classification tasks.

The most popular way to adjust the free parameters (the strength or weight of the synapses), in order to teach the neural network to accomplish the desired task, is the error backpropagation algorithm by Rumelhart et al. (1986), which consists of exploring the error hypersurface by following the reversed local error gradient. By presenting the network with a series of examples for which known desired outputs are available (the training set), the local gradient of the total error with respect to the connection weights can be computed and the weights correspondingly updated. There are several techniques to achieve generalization, understood as the ability of a network to imprint in its weights the abstract rules for classification implicit in the training examples, disregarding at the same time the particular details of the examples used. Again, the most common practice consists of dividing the available set of examples into three groups: a training set, a validation set and a test set. Learning proceeds by minimizing the training set error while at the same time monitoring the validation error. When the network has captured the general rules for classification and started to incorporate the particular details of the training set, the validation error reaches a minimum while the training error continues decreasing. It is at this minimum point that learning is stopped, in order to avoid overtraining, and the error of the network is estimated using the error set. There are multiple variations to this very basic scheme, but most of them end up in the vicinity of a local minimum of the error hypersurface which we expect to be the global minimum.

Here we deviate from the common practice and use a different formalism, which we consider more flexible and sound: Bayesian training of neural networks. In the Bayesian framework, instead of a class assignement we obtain a predictive probability distribution. Let $\boldsymbol{\theta}$ denote the set of parameters needed to fully specify a neural network architecture (i.e., all the connection weights between neurons in the network). The network class prediction $C_{n+1}$ for a new test case $\boldsymbol{x}_{n+1}$ given a training set $\mathcal{S}_{\text {train }}$ is computed as

$P\left(C_{n+1} \mid \boldsymbol{x}_{n+1}, \mathcal{S}_{\text {train }}\right)=\int P\left(C_{n+1} \mid \boldsymbol{x}_{n+1}, \boldsymbol{\theta}\right) \cdot P\left(\boldsymbol{\theta} \mid \mathcal{S}_{\text {train }}\right) \cdot \mathrm{d} \boldsymbol{\theta}$,

that is, an average of the predictions $P\left(C_{n+1} \mid \boldsymbol{x}_{n+1}, \boldsymbol{\theta}\right)$ made by networks covering the whole $\boldsymbol{\theta}$ parameter space, weighted by the posterior probability of $\boldsymbol{\theta}$ given the training set. The expression $P\left(C_{n+1} \mid \boldsymbol{x}_{n+1}, \mathcal{S}_{\text {train }}\right)$ is a probability distribution for all possible classes to which $\boldsymbol{x}_{n+1}$ can be assigned, or, equivalently, for all possible values of $C_{n+1}$. The a posteriori probability can be computed by applying Bayes theorem

$P\left(\boldsymbol{\theta} \mid \boldsymbol{S}_{\text {train }}\right) \propto P\left(\boldsymbol{S}_{\text {train }} \mid \boldsymbol{\theta}\right) \cdot P(\boldsymbol{\theta})$,

that is, as the product of the likelihood function and the prior probability of the network parameters. Once this probability distribution is obtained, single value predictions can be obtained by minimization of loss functions, such as squared error loss (equivalent to guessing the mean) or absolute error loss (equivalent to guessing the median) or, as in our case, 0-1 loss functions more suitable for classification tasks (equivalent to guessing the mode). The integral in Eq. (1) is defined over all parameter space. In the case of neural networks, this integral is unmanageable without the aid of special techniques developed for solving similar problems in the context of theoretical physics. In this work we make use of hybrid Monte Carlo techniques implemented in the software package Flexible Bayesian Methods by Neal (1996). These are used to approximate the integral by a sum of terms of the form $P\left(C_{n+1} \mid \boldsymbol{x}_{n+1}, \boldsymbol{\theta}^{(n)}\right)$, where all the sets of weights $\boldsymbol{\theta}^{(n)}$ follow the probability distribution $P\left(\boldsymbol{\theta} \mid \mathcal{S}_{\text {train }}\right)$. The likelihood function $P\left(\mathcal{S}_{\text {train }} \mid \boldsymbol{\theta}\right)$ can be a Gaussian function for regression networks which incorporate noise in the width of the gaussian, or a softmax model for classification purposes. A full description of Markov Chain MonteCarlo (MCMC) techniques is clearly beyond the scope of this paper. We refer the interested reader to classical expositions of the method, such as Neal (1996) or Bishop (1995). We mention here that the method achieves equilibrium in the target statistical distribution only after a certain number of networks $N_{\text {eq }}$ have been generated. Therefore, in general, only networks created after $N_{\text {eq }}$ are used to estimate the integral.

This approach presents several advantages over traditional maximum likelihood methods such as error backpropagation. The main advantage stems from the fact that predictions are not formulated in terms of a unique network but as an average over all networks. Those networks with larger a posteriori probabilities contribute more to the average than the rest implying that it is no longer necessary to limit the model complexity.

As mentioned above, in classical backpropagation training, model complexity is usually limited in order to avoid overtraining because complex models can incorporate increasingly complex features of the input space, including random noise in the training set. For each training set size and statistical distribution of patterns there is an optimal model complexity that is usually sought by cross-validating the training performance with an independent set of examples called a validation set. By 
stopping the learning algorithm at the minimum of the validation error curve, we are effectively limiting the average norm of the weight vectors, thus limiting model complexity (see e.g. Bishop 1995). This is at the expense of reducing the available set of training examples to create the validation set. In the Bayesian framework, on the contrary, if the model and prior probabilities (henceforth priors) are appropriate, the inferences are right independent of the training set size, thus eliminating the need for cross validation and for the reduction of the training set implied by it.

One of the main advantages of Bayesian training of neural networks is the possibility of includincluding hierarchical priors in Eq. (2). It allows automatic relevance determination (ARD) of the parameters by introducing correlations amongst groups of parameters, in particular, amongst the set of weights connecting a given input unit with neurons in the next layer. A prior specification for the network parameters $\theta$ can be expressed as the product of several independent fully specified probabilities (one for each connection weight at the lowest level) or as the integral of a more general probability distribution that applies to the connection weights of a given unit and that is characterized by new sets of parameters. Because these newly introduced parameters directly dictate not the weight probability density but the probability distribution of the parameters that describe it (i.e., that describe the weight probability density), they are called hyperparameters. In the first case, a Gaussian prior with fixed mean and width can be used directly for the probability distribution of a given connection weight. In the second approach, this probability distribution of weights in the network would be the result of averaging over all possible means and widths (hyperparameters) weighted by their respective prior probabilities:

$$
P(\boldsymbol{\theta})=\int P(\boldsymbol{\theta} \mid \gamma) \cdot P(\gamma)
$$

where, in the example, $\gamma$ is the vector of hyperparametric means and/or widths that is common for all synaptic weights out of the neuron. $P(\gamma)$ in turn can be fully specified or else given in terms of new hyperparameters at the next level of neural units in the same layer. By using different levels of hyperparameters from the bottom levels of single connection weights or single unit weight sets up to the highest level of layers or the entire network, correlations amongst parameter sets of the same group can be introduced in the integral of Eq. (1).

This scheme, when applied to the input-hidden connection weights, can be used to test the relevance of input variables for the classification task. If a given input variable is not relevant for classification purposes, under very special circumstances it may worsen the network performance. By making use of these hierarchical priors, we can effectively remove noninformative input units simply because a large fraction of the parameter space with significant contributions to the integral on the right hand side of Eq. (1) will come from networks with their connection weights set to zero.

\subsection{Preprocessing of Hipparcos training patterns}

We have used light curves from the Hipparcos catalogue as training set. As usual with neural networks, the raw data (originally in the JD- $V$ magnitude space) need to be preprocessed to optimize the performance of the network. The preprocessing of light curves consists of several distinct stages briefly summarized here to serve as a guide for the following explanations.

1. Unit conversion and binning.

2. Pattern completion.

(a) pattern regression;

(b) normalization;

(c) SOM consultation;

(d) second order interpolation;

3. Pattern regression.

4. Normalization and phase-shifting.

\subsubsection{Unit conversion and binning}

First, original JD $-V$ magnitude light curves from the Hipparcos archive are extracted and observations with bad quality flags removed. Then, the time coordinate is converted into phase according to Hipparcos ephemeris, if available. Otherwise, literature ephemerides provided with the catalogue are used. The resulting light curve is binned into 50 phase intervals between 0 and 1 corresponding to $\Delta \phi=0.02$. In a high fraction of the catalogue, light curves contain gaps in the phase coverage of the binary cycle.

The analysis in terms of the information content (IC) of the inputs supports the choice of 50 bins as a compromise between maximum possible resolution with manageable sizes. As a rule, very narrow phase bins can preserve finer details of the light curve. In practice however, there is a limit above which fine details convey no useful information for the classification task. We found that the smallest detail necessary for the classification task in our classification scheme was given by the eccentricity definition (see Sect. 3.3). At a resolution of $\Delta \phi=0.02$, a system is classified as eccentric if the secondary eclipse is more than two bins/input units away from phase $\phi=0.25$.

Bearing that in mind, we studied the information content distribution along the light curve. We defined the information content of a given light curve as the sum of the mutual information content of the measured phase bins and the class. The mutual information $I$ between two random variables $X$ and $Y$ was defined as

$I(X, Y)=\sum_{x, y} p(x, y) \log _{2} \frac{p(x, y)}{p(x) \cdot p(y)}$.

We have computed the mutual information between each of the 50 phase bins $\left(X_{i}, 1<i<50\right)$ and the class category $(Y)$. The resulting distribution is shown in Fig. 6. Equivalent plots at higher resolutions (i.e., with smaller $\Delta \phi$ ) do not change this smooth curve.

This plot shows that at a resolution of 50 bins, the mutual information curve is smooth and intuitively reflects the knowledge needed by a human classifier. At much higher resolutions (which do not convey more information), there are not 


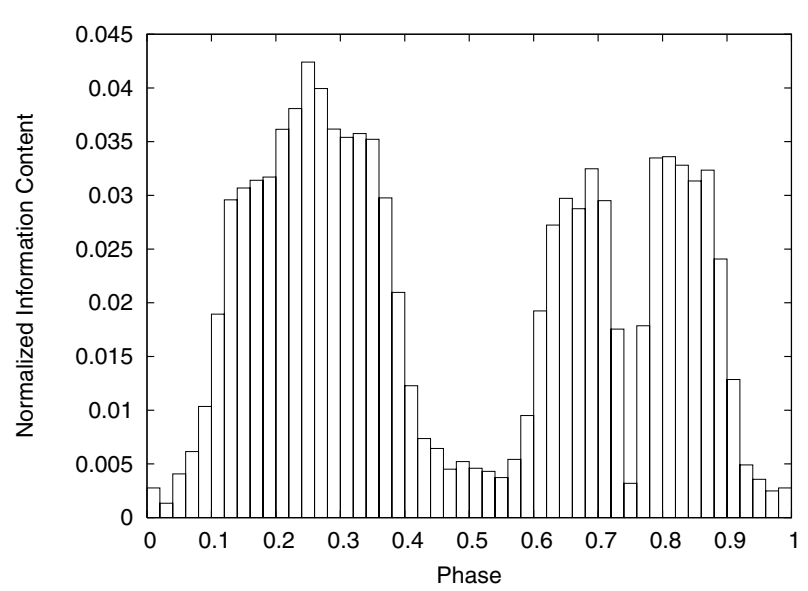

Fig. 6. Normalized mutual information between each of the phase bins and the class.

enough examples to characterize the relationship between each bin mean and the class, and the performance of the neural networks degrades. At lower resolutions, important information is lost and, again, the performance degrades, especially when identifying eccentricity.

\subsubsection{Pattern completion}

Although neural networks are characterized by a high fault tolerance that can be assimilated to the presence of gaps in the phase coverage, we observed improved performance of the classification network when these gaps are interpolated according to the procedure presented below, mainly in cases where incomplete phase coverage is worsened by the presence of significant noise and/or outliers. Therefore, chose to interpolate data in the gaps by applying the following approach. The incomplete light curve is presented to a Self Organized Kohonen Map (SOM) constructed with the best and most representative 721 light curves of the Hipparcos catalogue, including pulsating variables (see below). These were chosen to cover as many morphological features as possible with low noise levels and a phase coverage such that a simple spline interpolation allows reliable recovery of all morphological information. Following presentation of the incomplete light curve to the SOM, the map of neural activity is searched for the most similar light curve of the map that uses the Euclidean distance criterion.

The SOM was created using the standard software package SOM_PAK prepared by the SOM programming team of the Helsinki University of Technology. The map had dimensions 10 by 8 , it was trained 100 times with different initializations, and the lowest quatization error map was saved for subsequent use. During training, Gaussian neighbourhood functions were used. The automatic procedure implemented in SOM_PAK for the search of such minimum error nets implies a random initialization and two training stages: during the first 1000 cycles, unit vectors are ordered in a process whereby the neighbourhood radius decreases from values close to the map size down to unity, and the learning rate decreases from 0.05 to zero. During the next 10000 cycles, unit vectors are fine-tuned to minimize quantization error by training with smaller rates (starting at 0.02) and neighbourhood radii (starting from 3.0). The choice of the map dimensions is justified in terms of the Sammon mapping of the input set (Sammon 1969).

Presentation of an incomplete light curve to the SOM requires adequate preprocessing. In this case, the preprocesing consists of normalizing the light curve considered as a 50-component vector to unit length, as done with the map creation vectors. The reason for this is that a SOM operates by computing the scalar product of the input vector and each of the neural codebook vectors, thus constituting a morphological similarity detector. Therefore, it is necessary to scale the input's incomplete light curve vector to a length at least close to the one used for the codebook vectors. Unfortunately, the normalization constant of an incomplete light curve will be smaller than if it were complete, by a factor that depends on the gap total length and the precise phases missing from the curve. Thus, in order to properly normalize the incomplete light curve, we need the same phase bins that we want to retrieve from the SOM. To overcome this difficulty, the original data previous to the phase-binning process are regressed using a set of neural networks obtained under the Bayesian framework described above.

The regression network is indeed a set of networks, the parameters of which follow the distribution function $P\left(\boldsymbol{\theta} \mid \mathcal{S}_{\text {train }}\right)$, with $\mathcal{S}_{\text {train }}$ the set of points in the light curve. This set of networks is generated by specifying priors with hyperparameters for input-to-hidden weights, hidden biases, hidden-to-output weights, and output biases.

The prior specification used for the output bias is a Gaussian prior with a mean of zero and standard deviation 10. For input-to-hidden weights and hidden biases, a Gaussian distribution is used with zero mean and variance given by a gamma distribution of mean equal to 2.0 and $\alpha=0.5$, where alpha is the shape parameter. Finally, the hidden-to-output weights are given Gaussian priors with mean equal to 3.0 and $\alpha=0.5$. These weights are automatically rescaled based on the number of hidden units so that the effect is independent of the hidden layer size in the limit of large numbers. Again, we refer those readers interested in the details of this method to the classical exposition by Neal (1996).

This regression network is then used to interpolate the missing gaps and the result is used as the basis for computing the normalization constant. It is important to notice that the regression is only used for normalization purposes. The query to the SOM is made with the incomplete light curve, previously normalized to unity with the length derived from the light curve completed by regression.

Once the SOM has been consulted and the resulting neural activity map searched for the closest exemplars, these are used to fill in the gaps of the incomplete input. The process modifies the zero and first-order terms of the retrieved exemplar in the missing phase interval (i.e., adds a linear function) to match the limiting data, and it uses only higher order curvature terms. The correction applied to the values of the retrieved exemplars in the phase gaps are given by

$V_{i}=V_{i}^{\mathrm{ex}} \cdot\left(\alpha+m \cdot\left(\phi_{i 2}-\phi_{i 1}\right)\right)$ 
L. M. Sarro et al.: Automatic classification of light curves, Online Material p 5
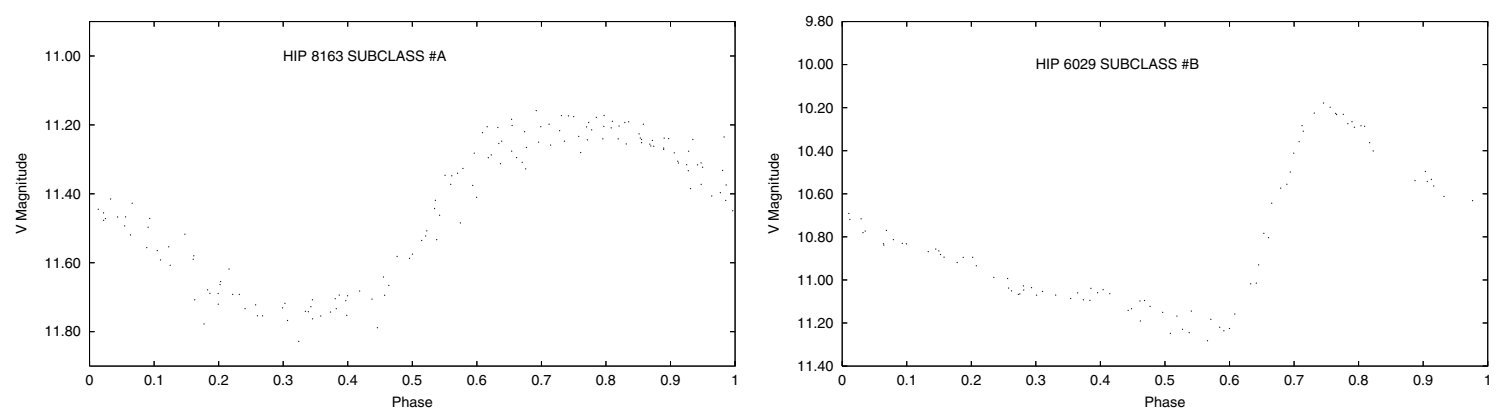

Fig. 7. Two examples of the new classes used to separate pulsating star light curves from binary systems.

where

$m=(\beta-\alpha) /\left(\phi_{i 2}-\phi_{i 1}\right)$

$\alpha=V_{i 1} / V_{i 1}^{\text {ex }}$

$\beta=V_{i 2} / V_{i 2}^{\mathrm{ex}}$,

and $i 1$ is the subindex of the last sampled phase bin before the gap, $i 2$ is the subindex of the first sampled phase bin after the gap, $V_{i 1}$ and $V_{i 2}$ are the values of the average magnitudes measured in the corresponding phase bins of the incomplete curve, and $V_{i 1}^{\text {ex }}$ and $V_{i 2}^{\text {ex }}$ the magnitudes in bins $i 1$ and $i 2$ of the exemplar curve.

\subsubsection{Pattern regression}

The result of the SOM-based pattern completion ( $V$ magnitudes for phase bins not sampled) is added to original data obtained from the catalogue before binning in phase. The completed light curve is regressed again using a second set of neural networks totally equivalent to the one used in the pattern completion stage, and the result is used to obtain an equispaced light curve of, again, 50 bins.

\subsubsection{Normalization and phase-shifting}

Finally, the result is shifted in phase, to make the minimum of the light curve (maximum magnitude) coincide with $\phi=0.75$, and rescaled in magnitude to match the $[0,1]$ interval. This final product is used both in the training of the classification network and as input to the trained classifier.

\subsection{Classification}

A total number of 1722 light curves were used for the training of the network. The relative size of each group of curves is given in Table 1 . In it, we split the set of type 1 light curves to create a new group of systems (named type 0 ) with essentially the same detached configuration made explicit in Sect. 2, but with $\Delta \phi$ between maxima greater than 0.29 or less than 0.21 , i.e., systems with clearly detectable eccentricies with the phase bin width used in the preprocessing. Of the 1722, 1610 were directly taken from the Hipparcos catalogue, while the remaining 112 are synthetic light curves of eccentric systems covering all possible $\Delta \phi$ between eclipses in steps of one phase bin, and depths of the secondary eclipse relative to the primary of 100 ,
80, 60, and 40\%. This addition to the basic Hipparcos training set was included to improve the poor performance of the classifier as an eccentricity detector when trained only with Hipparcos light curves, basically due to the scarcity of eccentric binary systems. Given that eccentric binaries only represent a small percentage of the total 1610 light curves, the overall performance of the network did not improve (it even degraded from a $6.1 \%$ average misclassification rate to $6.9 \%$ ), but the misclassification rate that was restricted to the eccentric systems lowered from an average $20 \%$ down to $4 \%$. The advantage introduced by the new class of eccentric systems is the possibility of using this classifier as a first stage in the automatic generation of lists of pre-main sequence binary system candidates in which the light curve information is combined with spectral or colour data.

Besides splitting type 1 systems, the table includes two new groups of light curves corresponding to pulsating variables light curves of two morphologies: sine-like curves with symmetric ascending and descending slopes (type A) and asymmetric light curves (type B). Figure 7 shows light curves from the Hipparcos catalogue as examples of both new types of pulsating morphologies. The reason for this noninformative classification is that morphological information alone is not enough to separate the different classes of pulsating stars. As mentioned above, this is the subject of ongoing research. The exact statistics of the pulsating stars light curves used for training are given in Table 2.

In this case, the output bias was given a Gaussian distribution of zero mean and variance given by a hyperparameter taken from a gamma distribution of mean 0.05 and shape parameter 0.5 . This was also the case for the (rescaled) hidden-tooutput weights and the hidden biases. Input-to-hidden weights were given a higher level of hyperparameters: their values were taken from a Gaussian distribution of zero mean and a hyperparameterized variance; this variance in turn was given for all such weights of a given input unit by a gamma distribution of shape parameter 0.5 and mean given by the overall gamma distribution (common for all input units) of mean 0.2 and shape parameter 0.5 . This hierarchical scheme introduces dependencies in the values of the weights connecting a given input unit to the hidden layer, thus allowing automatic (implicit) pruning of the input unit, if it does not add a significant improvement to the performance of the net. Finally as mentioned above, a softmax model is used in which the probability that the input 
L. M. Sarro et al.: Automatic classification of light curves, Online Material p 6

light curve $\boldsymbol{x}$ is of class $k$ is defined in terms of the output of the network as

$P\left(C=C_{k} \mid \boldsymbol{x}\right)=\frac{\exp \left(f_{k}(\boldsymbol{x})\right)}{\sum_{k^{\prime}} f_{k^{\prime}}(\boldsymbol{x})}$,

with $f_{k^{\prime}}$ the output of neuron $k^{\prime}$ that represents class $C_{k^{\prime}}$.
The integral in Eq. (1) is approximated by the sum of 200 terms generated by the Markov Chain MonteCarlo method after the distribution is let stabilize in 800 initial steps. Visual (9) inspection of the evolution of the error and weights confirms that, even before 600 initial steps, the method attains an equilibrium distribution. The errors reported in the next section have the same statistical properties if computed with the last 300 or 400 networks generated. 
L. M. Sarro et al.: Automatic classification of light curves, Online Material $p 7$

Table 4. Relevant physical parameters of systems classified as class 1 in the text.

\begin{tabular}{|c|c|c|c|c|c|c|c|c|c|c|}
\hline $\begin{array}{l}\text { Name } \\
\text { HD }\end{array}$ & $\begin{array}{l}P(\mathrm{~d}) \\
V_{\max }\end{array}$ & $\begin{array}{l}q \\
a\left(R_{\odot}\right)\end{array}$ & Comp. & $\begin{array}{l}\text { Spec. } \\
\text { type }\end{array}$ & $\begin{array}{l}M \\
\left(M_{\odot}\right)\end{array}$ & $\begin{array}{l}R \\
\left(R_{\odot}\right)\end{array}$ & $\begin{array}{l}T_{\text {eff }} \\
(\mathrm{K})\end{array}$ & $\begin{array}{l}\log (L) \\
L\left({ }_{\odot}\right)\end{array}$ & $\overline{M_{V}}$ & Ref. \\
\hline BW Aqr & 6.72 & 0.931 & $\overline{\mathrm{A}}$ & F7V & 1.488 & 2.064 & 3.803 & 0.79 & 2.74 & 1,2 \\
\hline $\mathrm{BD}-16^{\circ} 6074$ & 10.33 & 21.298 & B & F5V & 1.386 & 1.788 & 3.810 & 0.70 & 2.98 & \\
\hline V539 Ara & 3.17 & 0.851 & $\bar{A}$ & $\mathrm{~B} 3 \mathrm{~V}$ & 6.254 & 4.432 & 4.260 & 3.29 & -1.70 & $1,3,4,5$ \\
\hline HD 161783 & 5.71 & 20.53 & B & B $4 \mathrm{~V}$ & 5.326 & 3.734 & 4.230 & 3.02 & -1.04 & \\
\hline EM Car & 3.41 & 0.936 & $\bar{A}$ & $08 \mathrm{~V}$ & 22.89 & 9.34 & 4.531 & 5.02 & 4.56 & 1,6 \\
\hline HD 97484 & 8.38 & 33.72 & B & $08 \mathrm{~V}$ & 21.43 & 8.33 & 4.531 & 4.92 & 4.31 & \\
\hline GL Car & 2.42 & 0.962 & $\bar{A}$ & B8V & 13.5 & 4.99 & 4.476 & 5.02 & -2.97 & 7 \\
\hline HD 306168 & 8.38 & 22.60 & $\mathrm{~B}$ & $\mathrm{~B} 8 \mathrm{~V}$ & 13.0 & 4.74 & 4.468 & 4.92 & -2.83 & \\
\hline QX Car & 4.48 & 0.915 & $\mathrm{~A}$ & $\mathrm{~B} 2 \mathrm{~V}$ & 9.267 & 4.289 & 4.377 & 3.72 & -2.32 & $1,4,8$ \\
\hline HD 86118 & 6.64 & 29.81 & B & $\mathrm{B} 2 \mathrm{~V}$ & 8.480 & 4.051 & 4.354 & 3.58 & -2.07 & \\
\hline SZ Cen & 4.11 & 0.982 & $\bar{A}$ & A7V & 2.317 & 4.554 & 3.875 & 1.77 & 0.29 & $1,9,10$ \\
\hline HD 120359 & 8.48 & 17.94 & $\mathrm{~B}$ & $\mathrm{~A} 7 \mathrm{~V}$ & 2.277 & 3.624 & 3.892 & 1.64 & 0.61 & \\
\hline CW Cep & 2.73 & 0.893 & $\mathrm{~A}$ & $\mathrm{BO} .5 \mathrm{~V}$ & 13.52 & 5.685 & 4.452 & 4.27 & -3.17 & $1,11,12$ \\
\hline HD 218066 & 7.59 & 24.217 & $\mathrm{~B}$ & BO.5V & 12.08 & 5.177 & 4.442 & 4.15 & -2.94 & \\
\hline EK Cep & 4.43 & 0.553 & $\overline{\mathrm{A}}$ & $\mathrm{Al} .5 \mathrm{~V}$ & 2.029 & 1.579 & 3.954 & 1.17 & 1.89 & $1,11,13,14,15$ \\
\hline HD 206821 & 7.87 & 16.63 & $\mathrm{~B}$ & G5Vp & 1.124 & 1.315 & 3.756 & 0.21 & 4.31 & \\
\hline RZ Cha & 2.83 & 0.994 & $\mathrm{~A}$ & F5V & 1.518 & 2.264 & 3.810 & 0.90 & 2.46 & $1,10,16$ \\
\hline HD 93486 & 8.10 & 12.17 & $\mathrm{~B}$ & F5V & 1.509 & 2.264 & 3.810 & 0.90 & 2.46 & \\
\hline V442 Cyg & 2.39 & 0.901 & $\mathrm{~A}$ & FIV & 1.564 & 2.072 & 3.839 & 0.94 & 2.35 & 1,17 \\
\hline HD 334426 & 9.72 & 10.81 & B & $\mathrm{F} 2 \mathrm{~V}$ & 1.410 & 1.662 & 3.833 & 0.72 & 2.89 & \\
\hline V1143 Cyg & 7.64 & 0.968 & $\mathrm{~A}$ & F5V & 1.391 & 1.346 & 3.810 & 0.45 & 3.60 & $1,10,11$ \\
\hline HD 185912 & 5.86 & 22.82 & B & F5V & 1.347 & 1.323 & 3.806 & 0.42 & 3.67 & \\
\hline DI Her & 10.55 & 0.874 & $\mathrm{~A}$ & B5V & 5.185 & 2.680 & 4.230 & 2.73 & -0.46 & 1,18 \\
\hline HD 175227 & 8.42 & 43.18 & $\mathrm{~B}$ & B5V & 4.534 & 2.477 & 4.179 & 2.46 & 4.05 & \\
\hline RX Her & 1.78 & 0.847 & $\bar{A}$ & B9 & 2.75 & 2.44 & 4.015 & 1.79 & 0.48 & $10,11,19$ \\
\hline HD 170757 & 7.26 & 10.62 & $\mathrm{~B}$ & A0 & 2.33 & 1.96 & 3.985 & 1.48 & 1.12 & \\
\hline Al Hya & 8.29 & 0.922 & $\mathrm{~A}$ & $\mathrm{~F} 2 \mathrm{~m}$ & 2.145 & 3.914 & 3.826 & 1.44 & 1.10 & 1,20 \\
\hline $\mathrm{BD}+0^{\circ} 2259$ & 9.36 & 27.630 & $\mathrm{~B}$ & FOV & 1.978 & 3.850 & 3.851 & 1.24 & 1.61 & \\
\hline TZ Men & 8.57 & 0.604 & $\mathrm{~A}$ & $\mathrm{~A} 0 \mathrm{~V}$ & 2.487 & 2.016 & 4.017 & 1.63 & 0.93 & 1,21 \\
\hline HD 39780 & 6.19 & 27.94 & $\mathrm{~B}$ & A8V & 1.504 & 1.432 & 3.857 & 0.69 & 2.97 & \\
\hline UX Men & 4.18 & 0.967 & $\bar{A}$ & F5V & 1.238 & 1.347 & 3.792 & 0.38 & 3.81 & $10,22,23$ \\
\hline HD 37513 & 8.22 & 14.68 & $\mathrm{~B}$ & F8V & 1.198 & 1.274 & 3.789 & 0.32 & 3.96 & \\
\hline V451 Oph & 2.20 & 0.848 & $\overline{\mathrm{A}}$ & B9V & 2.776 & 2.640 & 4.033 & 1.93 & 0.34 & $1,10,11,24$ \\
\hline HD 170470 & 7.87 & 12.27 & $\mathrm{~B}$ & $\mathrm{~A} 0 \mathrm{~V}$ & 2.356 & 2.028 & 3.991 & 1.53 & 1.11 & \\
\hline V1031 Ori & 3.41 & 0.936 & $\mathrm{~A}$ & A6V & 2.473 & 4.321 & 3.895 & 1.80 & 0.18 & 1,25 \\
\hline HD 38735 & 6.02 & 33.727 & $\mathrm{~B}$ & $\mathrm{~A} 3 \mathrm{~V}$ & 2.286 & 2.977 & 3.924 & 1.60 & 0.74 & \\
\hline Al Phe & 24.59 & 0.966 & $\mathrm{~A}$ & KOIV & 1.236 & 2.930 & 3.700 & 0.69 & 3.24 & 1,26 \\
\hline HD 6980 & 8.61 & 47.830 & $\mathrm{~B}$ & F7V & 1.195 & 1.816 & 3.800 & 0.67 & 3.07 & \\
\hline Zeta Phe & 1.67 & 0.649 & A & B6V & 3.930 & 2.851 & 4.163 & 2.51 & 4.59 & $1,3,10,27$ \\
\hline HD 6882 & 3.95 & 11.039 & $\mathrm{~B}$ & $\mathrm{~B} 8 \mathrm{~V}$ & 2.551 & 1.853 & 4.076 & 1.79 & 0.91 & \\
\hline V1647 Sgr & 3.28 & 0.900 & $\mathrm{~A}$ & AIV & 2.189 & 1.831 & 3.982 & 1.41 & 1.35 & 1,28 \\
\hline HD 163708 & 6.94 & 14.93 & $\mathrm{~B}$ & AIV & 1.972 & 1.666 & 3.959 & 1.23 & 1.73 & \\
\hline V760 Sco & 1.73 & 0.927 & $\mathrm{~A}$ & B4V & 4.980 & 3.013 & 4.228 & 2.82 & 4.71 & 1,29 \\
\hline HD 147683 & 6.99 & 12.88 & $\mathrm{~B}$ & $\mathrm{~B} 4 \mathrm{~V}$ & 4.620 & 2.640 & 4.210 & 2.63 & 4.24 & \\
\hline CV Vel & 6.89 & 0.982 & $\bar{A}$ & $\mathrm{~B} 2.5 \mathrm{~V}$ & 6.100 & 4.087 & 4.253 & 3.19 & -1.48 & 1,30 \\
\hline HD 77464 & 6.69 & 34.96 & B & $\mathrm{B} 2.5 \mathrm{~V}$ & 5.996 & 3.948 & 4.250 & 3.15 & -1.38 & \\
\hline
\end{tabular}

References: 1: Andersen (1991), 2: Clausen (1991), 3: Andersen (1983), 4: De Greve (1989), 5: Clausen (1996), 6: Andersen \& Clausen (1989), 7: Giménez \& Clausen (1986), 8: Andersen et al. (1983b), 9: Gronbech et al. (1977), 10: Popper (1980), 11: Fracastoro (1972), 12: Clausen \& Giménez (1991), 13: Popper (1987), 14: Martín \& Rebolo (1993), 15: Claret et al. (1995), 16: Jorgensen \& Gyldenkerne (1975), 17: Lacy \& Frueh (1987), 18: Popper (1982), 19: Jeffreys (1980), 20: Khaliullin \& Kozyreva (1989), 21: Andersen et al. (1987), 22: Clausen \& Gronbech (1976), 23: Andersen et al. (1989), 24: Clausen et al. (1986), 25: Andersen et al. (1990), 26: Andersen et al. (1988), 27: Clausen et al. (1976), 28: Clausen et al. (1977), 29: Andersen et al. (1985), 30: Clausen \& Gronbech (1977). 
L. M. Sarro et al.: Automatic classification of light curves, Online Material $p 8$

Table 5. Relevant physical parameters of systems classified as class 2 in the text.

\begin{tabular}{|c|c|c|c|c|c|c|c|c|c|c|}
\hline $\begin{array}{l}\text { Name } \\
\text { HD }\end{array}$ & $\begin{array}{l}P(\mathrm{~d}) \\
V_{\max } \\
\end{array}$ & $\begin{array}{l}q \\
a\left(R_{\odot}\right) \\
\end{array}$ & Comp. & $\begin{array}{l}\text { Spec. } \\
\text { type }\end{array}$ & $\begin{array}{l}M \\
\left(M_{\odot}\right) \\
\end{array}$ & $\begin{array}{l}R \\
\left(R_{\odot}\right) \\
\end{array}$ & $\begin{array}{l}T_{\text {eff }} \\
(\mathrm{K}) \\
\end{array}$ & $\begin{array}{l}\log (L) \\
L\left(_{\odot}\right) \\
\end{array}$ & $M_{V}$ & Ref. \\
\hline RY Aqr & 1.9666 & 0.204 & $\mathrm{~A}$ & A3 & 1.27 & 1.28 & 3.881 & 0.700 & 2.9 & 1,2 \\
\hline HD 203069 & 9.06 & 7.61 & B & & 0.26 & 1.79 & 3.658 & 0.100 & 4.4 & \\
\hline IM Aur & 1.2473 & 0.249 & $\mathrm{~A}$ & B9 & 4.73 & 3.24 & 4.199 & 2.770 & -2.2 & $1,3,4$ \\
\hline HD 33853 & 7.70 & 8.81 & B & & 1.18 & 2.20 & 3.881 & 1.160 & 1.8 & \\
\hline $\mathrm{R} \mathrm{CMa}$ & 1.1359 & 0.131 & A & $\mathrm{F} 2 \mathrm{~V}$ & 1.52 & 1.73 & 3.849 & 0.76 & 2.77 & 3,5 \\
\hline HD 57167 & 4.5730 & 5.48 & $\mathrm{~B}$ & G8IV & 0.20 & 1.18 & 3.712 & -0.41 & 6.36 & \\
\hline RZ Cas & 1.195 & 0.330 & $\mathrm{~A}$ & $\mathrm{~A} 3 \mathrm{~V}$ & 2.21 & 1.67 & 3.934 & 1.12 & & $3,6,7$ \\
\hline HD 17138 & 6.2 & 6.79 & B & & 0.73 & 1.94 & 3.672 & 0.16 & & \\
\hline TV Cas & 1.8126 & 0.464 & $\mathrm{~A}$ & B9V & 2.80 & 2.81 & 4.029 & 1.970 & -0.2 & $1,3,7,8$ \\
\hline HD 1486 & 10.57 & 10.01 & B & G5-9IV & 1.30 & 3.15 & 3.708 & 0.780 & 2.7 & \\
\hline $\mathrm{U} \mathrm{CrB}$ & 3.4522 & 0.300 & $\mathrm{~A}$ & B6V & 4.70 & 2.60 & 4.185 & 2.520 & -1.6 & $1,3,7,9,10$ \\
\hline HD 136175 & 7.65 & 17.57 & $\mathrm{~B}$ & GOIII-IV & 1.41 & 4.91 & 3.764 & 1.390 & 1.2 & \\
\hline SW Cyg & 4.5730 & 0.220 & $\mathrm{~A}$ & $\mathrm{~A} 2 \mathrm{~V}$ & 2.27 & 2.43 & 3.957 & 1.550 & 0.8 & 11 \\
\hline HD 191240 & 9.30 & 16.28 & $\mathrm{~B}$ & KOIV & 0.50 & 4.15 & 3.690 & 0.950 & 2.3 & \\
\hline AF Gem & 1.2435 & 0.342 & $\mathrm{~A}$ & B9.5V & 3.37 & 2.61 & 4.00 & 1.78 & & 7,12 \\
\hline HD 210892 & 10.54 & 8.04 & $\mathrm{~B}$ & G0III-IV & 1.155 & 2.32 & 3.768 & 0.75 & & \\
\hline AQ Peg & 5.5485 & 0.256 & $\mathrm{~A}$ & A2 & 2.34 & 2.64 & 3.959 & 1.630 & 0.6 & 1,11 \\
\hline $\mathrm{BD}+12^{\circ} 4653$ & 10.39 & 18.887 & $\mathrm{~B}$ & & 0.60 & 4.89 & 3.644 & 0.910 & 2.4 & \\
\hline AT Peg & 1.1461 & 0.472 & $\mathrm{~A}$ & A4V & 2.22 & 1.86 & 3.924 & 1.19 & 1.76 & $1,3,7,13$ \\
\hline HD 210892 & 9.50 & 6.84 & $\mathrm{~B}$ & & 1.05 & 2.15 & 3.690 & 0.38 & 4.1 & \\
\hline AW Peg & 10.6225 & 0.160 & A & AlVe & 2.06 & 1.90 & 3.959 & 1.350 & 1.3 & $1,3,11,14$ \\
\hline HD 207956 & 7.40 & 27.18 & $\mathrm{~B}$ & F5IV & 0.33 & 6.12 & 3.602 & 0.930 & 2.4 & \\
\hline DM Per & 2.7277 & 0.314 & A & $\mathrm{B} 5 \mathrm{~V}$ & 5.82 & 3.96 & 4.202 & 2.960 & -2.6 & $1,3,15,16$ \\
\hline HD 14871 & 7.88 & 16.18 & $\mathrm{~B}$ & A5III & 1.83 & 4.60 & 3.914 & 1.940 & -0.1 & \\
\hline RY Per & 6.8636 & 0.281 & A & $\mathrm{B} 3 \mathrm{~V}$ & 6.60 & 4.00 & 4.246 & 3.140 & -3.2 & $1,17,18$ \\
\hline HD 17034 & 8.48 & 30.96 & B & F6IV & 1.86 & 8.53 & 3.814 & 2.070 & -0.5 & \\
\hline Bet Per & 2.8673 & 0.221 & $\mathrm{~A}$ & $\mathrm{~B} 8 \mathrm{~V}$ & 3.70 & 2.90 & 4.097 & 2.250 & -0.9 & $1,3,19,20$ \\
\hline HD 19356 & 2.12 & 14.04 & $\mathrm{~B}$ & G8-KOIII & 0.82 & 3.50 & 3.708 & 0.860 & 2.5 & \\
\hline U Sge & 3.3806 & 0.333 & $\mathrm{~A}$ & B8V & 5.70 & 4.05 & 4.146 & 2.750 & -2.2 & $1,9,18,21$ \\
\hline HD 181182 & 8.20 & 18.63 & $\mathrm{~B}$ & G4III & 1.90 & 5.38 & 3.724 & 1.310 & 1.4 & \\
\hline XZ Sgr & 3.2756 & 0.137 & $\mathrm{~A}$ & A3V & 1.89 & 1.46 & 3.945 & 1.060 & 2.0 & 1,22 \\
\hline HD 168710 & 0.00 & 11.98 & $\mathrm{~B}$ & G5IV & 0.26 & 2.47 & 3.708 & 0.570 & 3.3 & \\
\hline TX UMa & 3.2756 & 0.137 & $\mathrm{~A}$ & B8V & 4.76 & 2.83 & 4.111 & 2.30 & & $1,3,9,23$ \\
\hline HD 93033 & 7.06 & 11.98 & B & G0III-IV & 1.18 & 4.24 & 3.740 & 1.17 & & \\
\hline
\end{tabular}

References: 1: Giuricin et al. (1983), 2: Helt (1987), 3: Fracastoro (1972), 4: Gulmen et al. (1985), 5: Sarma et al. (1996), 6: Maxted et al. (1994), 7: Maxted \& Hilditch (1996), 8: Khalesseh \& Hill (1992), 9: Cester et al. (1977), 10: Van Gent (1989), 11: Wilson \& Mukherjee (1988), 12: Maxted \& Hilditch (1995), 13: Maxted et al. (1994), 14: Derman \& Demircan (1992), 15: Hilditch et al. (1986), 16: Hilditch et al. (1992), 17: Olson \& Plavec (1997), 18: Van Hamme \& Wilson (1986), 19: Richards et al. (1988), 20: Kim (1989), 21: Khan \& Budding (1986), 22: Knipe (1974), 23: Maxted et al. (1995). 
L. M. Sarro et al.: Automatic classification of light curves, Online Material $p 9$

Table 6. Relevant physical parameters of systems classified as class 3 in the text.

\begin{tabular}{|c|c|c|c|c|c|c|c|c|c|c|}
\hline $\begin{array}{l}\text { Name } \\
\text { HD }\end{array}$ & $\begin{array}{l}P(\mathrm{~d}) \\
V_{\max }\end{array}$ & $\begin{array}{l}q \\
a\left(R_{\odot}\right)\end{array}$ & Comp. & $\begin{array}{l}\text { Spec. } \\
\text { type }\end{array}$ & $\begin{array}{l}M \\
\left(M_{\odot}\right)\end{array}$ & $\begin{array}{l}R \\
\left(R_{\odot}\right)\end{array}$ & $\begin{array}{l}T_{\text {eff }} \\
(\mathrm{K})\end{array}$ & $\begin{array}{l}\log (L) \\
L\left(_{\odot}\right)\end{array}$ & $M_{V}$ & Ref. \\
\hline \multirow[t]{2}{*}{ CX Aqr } & 0.5559 & 0.537 & A & F5 & 1.19 & 1.29 & 3.806 & 2.70 & & 1,2 \\
\hline & 10.70 & 3.48 & B & G9 & 0.64 & 1.15 & 3.696 & 0.72 & & \\
\hline EE Aqr & 0.5089 & 0.322 & $\mathrm{~A}$ & F0-F2 & 2.20 & 1.75 & 3.881 & 0.88 & & $1,3,4$ \\
\hline HD 213683 & 8.30 & 3.83 & $\mathrm{~B}$ & & 0.71 & 1.07 & 3.643 & -0.42 & & \\
\hline IU Aur & 1.8115 & 0.676 & $\mathrm{~A}$ & $\mathrm{BOV}$ & 21.3 & 7.5 & 4.505 & 4.73 & -6.8 & $5,6,7$ \\
\hline HD 35652 & 8.19 & 20.58 & B & BO. $5 \mathrm{~V}$ & 14.4 & 7.2 & 4.449 & 4.46 & -6.3 & \\
\hline TT Aur & 1.3327 & 0.648 & $\mathrm{~A}$ & $\mathrm{~B} 2 \mathrm{~V}$ & 8.58 & 4.06 & 4.373 & 3.664 & -4.5 & 8,9 \\
\hline HD 33088 & 8.53 & 12.32 & B & & 5.56 & 4.17 & 4.267 & 3.264 & -3.5 & \\
\hline \multirow[t]{2}{*}{ DO Cas } & 0.6847 & 0.313 & $\mathrm{~A}$ & $\mathrm{~A}$ & 1.69 & 2.10 & 3.96 & 1.42 & & $1,5,10,11$ \\
\hline & 8.60 & 4.26 & $\mathrm{~B}$ & & 0.53 & 1.20 & 3.68 & -0.16 & & \\
\hline YY Cet & 0.79 & 0.510 & $\mathrm{~A}$ & A8 & 1.84 & 2.09 & 3.875 & 1.10 & & 1,12 \\
\hline $\mathrm{BD}-18^{\circ} 349$ & 5.05 & 10.00 & B & & 0.94 & 1.63 & 3.725 & 0.30 & & \\
\hline AI Cru & 1.4177 & 0.611 & A & B2IVe & 10.30 & 4.95 & 4.384 & 3.880 & -4.9 & 13 \\
\hline-60.3723 & 9.20 & 13.54 & B & B4 & 6.30 & 4.43 & 4.248 & 3.240 & -3.3 & \\
\hline V836 Cyg & 0.6534 & 0.333 & $\mathrm{~A}$ & A3 & 2.4 & 1.96 & 4.00 & 1.04 & & $1,10,14$ \\
\hline HD 203470 & 8.59 & 4.67 & $\mathrm{~B}$ & $\mathrm{G}$ & 0.80 & 1.24 & 3.76 & 4.32 & & \\
\hline \multirow[t]{2}{*}{ RZ Dra } & 0.5508 & 0.442 & $\mathrm{~A}$ & A5 & 1.40 & 1.62 & 3.911 & & & 1,15 \\
\hline & 10.00 & 3.57 & B & $\mathrm{K} 2$ & 0.62 & 1.12 & 3.690 & & & \\
\hline RU Eri & 0.6322 & 0.420 & $\mathrm{~A}$ & F3V & 2.45 & 2.06 & & 1.07 & & 1,10 \\
\hline HD 24658 & 9.90 & 4.69 & B & & 1.03 & 1.43 & & -0.03 & & \\
\hline $\mathrm{uHer}$ & 2.0510 & 0.38 & $\mathrm{~A}$ & B2IV & 7.60 & 5.80 & 4.301 & 3.680 & -4.5 & $5,6,16,17$ \\
\hline HD 156633 & 4.77 & 14.87 & B & B8III & 2.90 & 4.40 & 4.065 & 2.490 & -1.5 & \\
\hline TT Her & 0.9121 & 0.435 & $\mathrm{~A}$ & $\mathrm{~F} 2 \mathrm{~V}$ & 1.56 & 2.30 & 3.960 & 1.13 & & $1,10,18,19$ \\
\hline $\mathrm{BD}+17^{\circ} 3117$ & 5.17 & 9.70 & B & & 0.68 & 1.49 & 3.744 & -0.02 & & \\
\hline \multirow[t]{2}{*}{ RS Ind } & 0.6240 & 0.310 & A & F1V & 2.00 & 2.00 & 3.857 & 0.98 & & $1,3,20$ \\
\hline & 9.90 & 4.23 & B & G8 & 0.62 & 1.18 & 3.668 & -0.23 & & \\
\hline FT Lup & 0.470 & 0.426 & A & $\mathrm{F} 2 \mathrm{~V}$ & 1.43 & 1.43 & 3.826 & & & $1,21,22$ \\
\hline 132316 & 9.7 & 3.23 & B & $\mathrm{K} 5-7 \mathrm{~V}$ & 0.61 & 0.94 & 3.639 & & & \\
\hline V Pup & 1.4550 & 0.522 & $\mathrm{~A}$ & B1 & 14.86 & 6.18 & 4.450 & 4.340 & -6.1 & $5,6,23,24$ \\
\hline HD 65818 & 4.41 & 15.28 & B & B3 & 7.76 & 4.90 & 4.425 & 4.040 & -5.3 & \\
\hline CX Vir & 0.7461 & 0.336 & $\mathrm{~A}$ & F5 & 1.07 & 1.85 & & 0.75 & & 1,25 \\
\hline 123660 & 9.20 & 3.90 & B & $\mathrm{K}$ & 0.36 & 1.12 & & -0.31 & & \\
\hline
\end{tabular}

References: 1: Hilditch et al. (1988), 2: McFarlane et al. (1986a), 3: Hilditch \& King (1988), 4: Corvino et al. (1990), 5: Fracastoro (1972), 6: Giuricin et al. (1983), 7: Drechsel et al. (1994), 8: Wachmann et al. (1986), 9: Bell et al. (1987a), 10: Karimie \& Duerbeck (1985), 11: Barone et al. (1992), 12: McFarlane et al. (1986b), 13: Bell et al. (1987b), 14: Breinhorst et al. (1989), 15: Kreiner et al. (1994), 16: Cester et al. (1977) 17: Hilditch (1984), 18: Kwee \& Van Genderen (1983), 19: Milano et al. (1989), 20: Marton et al. (1990), 21: Hilditch et al. (1984), 22: Lipari \& Sistero (1986), 23: Schneider et al. (1979), 24: Andersen et al. (1983a), 25: Hilditch \& King (1988). 
Table 7. Relevant physical parameters of systems classified as class 4 in the text.

\begin{tabular}{|c|c|c|c|c|c|c|c|c|c|c|}
\hline $\begin{array}{l}\text { Name } \\
\text { HD }\end{array}$ & $\begin{array}{l}P(\mathrm{~d}) \\
V_{\max }\end{array}$ & $\begin{array}{l}q \\
a\left(R_{\odot}\right)\end{array}$ & Comp. & $\begin{array}{l}\text { Spec. } \\
\text { type }\end{array}$ & $\begin{array}{l}M \\
\left(M_{\odot}\right)\end{array}$ & $\begin{array}{l}R \\
\left(R_{\odot}\right)\end{array}$ & $\begin{array}{l}T_{\text {eff }} \\
(\mathrm{K})\end{array}$ & $\begin{array}{l}\log (L) \\
L\left(_{\odot}\right)\end{array}$ & $M_{V}$ & Ref. \\
\hline OO Aql & 0.507 & 0.888 & $\mathrm{~A}$ & G5V & 1.19 & 1.44 & 5700 & 1.97 & $1,2,3,4$ & \\
\hline HD 187183 & 9.20 & 4.570 & $\mathrm{~B}$ & & 1.34 & 1.00 & 5635 & 1.62 & & \\
\hline V535 Ara & 0.629 & 0.582 & $\mathrm{~A}$ & $\mathrm{~A} 8 \mathrm{~V}$ & 2.18 & 2.10 & 8750 & 3.17 & 1,5 & \\
\hline HD 159441 & 7.40 & 4.667 & $\mathrm{~B}$ & & 1.27 & 0.79 & 8572 & 7.86 & & \\
\hline $\mathrm{AO} \mathrm{Cam}$ & 0.329 & 0.766 & $\mathrm{~A}$ & & 1.03 & 0.98 & 5520 & 0.80 & 3,6 & \\
\hline $\mathrm{BD}+52^{\circ} 826$ & 9.50 & 2.452 & B & 0.88 & 0.79 & & 5826 & 0.80 & & \\
\hline \multirow[t]{2}{*}{ V523 Cas } & 0.233 & 0.569 & $\mathrm{~A}$ & K4 & 0.79 & 0.75 & 4207 & 0.16 & $3,7,8$ & \\
\hline & & 1.714 & B & & 0.58 & 0.45 & 4407 & 0.12 & & \\
\hline \multirow[t]{2}{*}{ V677 Cen } & 0.325 & 0.481 & $\mathrm{~A}$ & & 1.06 & 1.19 & 5745 & 1.39 & 3,6 & \\
\hline & 11.55 & 2.312 & $\mathrm{~B}$ & & 0.51 & 0.15 & 5841 & 0.27 & & \\
\hline V752 Cen & 0.370 & 0.575 & $\mathrm{~A}$ & F8V & 1.20 & 1.24 & 6210 & 2.06 & 3,6 & \\
\hline HD 101799 & & 2.681 & B & & 0.69 & 0.36 & 6234 & 0.65 & & \\
\hline VY Cet & 0.341 & 0.666 & $\mathrm{~A}$ & & 1.02 & 1.01 & 5393 & 0.77 & 9 & \\
\hline $\mathrm{BD}-20^{\circ} 345$ & 11.10 & 2.449 & B & & 0.83 & 0.68 & 5610 & 0.61 & & \\
\hline \multirow[t]{2}{*}{ CC Com } & 0.221 & 0.518 & $\mathrm{~A}$ & & 0.79 & 0.41 & 4302 & 0.17 & $2,3,10$ & \\
\hline & 11.00 & 1.634 & B & & 0.54 & 0.73 & 4500 & 0.11 & & \\
\hline \multirow[t]{2}{*}{ EK Com } & 0.267 & 0.580 & $\mathrm{~A}$ & & 0.93 & 0.92 & 5000 & 0.47 & 11 & \\
\hline & 12.70 & 1.981 & $\mathrm{~B}$ & & 0.54 & 0.28 & 5310 & 0.20 & & \\
\hline \multirow[t]{2}{*}{ FS Cra } & 0.264 & 0.755 & $\mathrm{~A}$ & & 0.86 & 0.82 & 4567 & 0.26 & 3,10 & \\
\hline & 13.80 & 1.984 & B & & 0.73 & 0.65 & 4700 & 0.23 & & \\
\hline YY Eri & 0.322 & 0.693 & $\mathrm{~A}$ & G5 & 1.01 & 1.02 & 5389 & 0.79 & $1,2,3,13$ & \\
\hline HD 26609 & 8.80 & 2.361 & B & & 0.70 & 0.44 & 5585 & 0.43 & & \\
\hline \multirow[t]{2}{*}{ SY Hor } & 0.312 & 0.659 & $\mathrm{~A}$ & & 0.97 & 0.95 & 4934 & 0.47 & 3,9 & \\
\hline & 11.40 & 2.266 & B & & 0.83 & 0.64 & 5240 & 0.47 & & \\
\hline V508 Oph & 0.345 & 0.527 & $\mathrm{~A}$ & G5 & 1.01 & 1.06 & & 0.087 & 14 & \\
\hline $\mathrm{BD}+13^{\circ} 3496$ & 10.00 & 2.444 & B & & 0.52 & 0.80 & & -0.286 & & \\
\hline \multirow{2}{*}{ BB Peg } & 0.362 & 0.405 & $\mathrm{~A}$ & F8 & 1.16 & 1.21 & 5883 & 1.58 & 3,15 & \\
\hline & 10.80 & 2.512 & B & & 0.78 & 0.47 & 6200 & 0.81 & & \\
\hline U Peg & 0.375 & 0.579 & $\mathrm{~A}$ & $\mathrm{G} 2 \mathrm{~V}$ & 1.33 & 1.28 & 5515 & 2.80 & $2,3,16$ & \\
\hline $\mathrm{BD}+15^{\circ} 4915$ & 9.70 & 2.800 & B & & 0.77 & 0.44 & 5800 & 1.28 & & \\
\hline AE Phe & 0.362 & 0.401 & $\mathrm{~A}$ & G1/G2V & 1.17 & 1.19 & 6000 & 1.63 & 3,13 & \\
\hline HD 9528 & 8.30 & 2.521 & B & & 0.79 & 0.47 & 6145 & 0.79 & & \\
\hline \multirow[t]{2}{*}{ YZ Phe } & 0.234 & 0.597 & $\mathrm{~A}$ & & 0.87 & 0.79 & 4800 & 0.30 & 17 & \\
\hline & 12.50 & 1.786 & B & & 0.52 & 0.35 & 5055 & 0.16 & & \\
\hline \multirow[t]{2}{*}{ FG Sct } & 0.271 & 0.781 & $\mathrm{~A}$ & & 0.87 & 0.73 & 4662 & 0.29 & 3,10 & \\
\hline & 13.70 & 2.036 & B & & 0.68 & 0.83 & 4800 & 0.25 & & \\
\hline RZ Tau & 0.416 & 0.369 & $\mathrm{~A}$ & A7V & 1.57 & 1.51 & 7200 & 5.51 & $1,2,18,19$ & \\
\hline HD 285892 & 10.50 & 3.024 & B & & 1.00 & 0.58 & 7146 & 2.34 & & \\
\hline \multirow[t]{2}{*}{ BP Vel } & 0.265 & 0.722 & $\mathrm{~A}$ & & 0.90 & 0.86 & 4717 & 0.33 & 20 & \\
\hline & 12.90 & 2.009 & B & & 0.65 & 0.48 & 5000 & 0.23 & & \\
\hline \multirow[t]{2}{*}{ BI Vul } & 0.252 & 0.686 & $\mathrm{~A}$ & & 0.86 & 0.82 & 4549 & 0.26 & 3,10 & \\
\hline & & 1.898 & B & & 0.70 & 0.59 & 4600 & 0.20 & & \\
\hline W UMa & 0.334 & 0.731 & $\mathrm{~A}$ & F8V:p & 1.08 & 1.10 & 5800 & 0.87 & $1,2,3$ & \\
\hline HD 83950 & 8.30 & 2.505 & B & & 0.79 & 0.51 & 6194 & 0.60 & & \\
\hline \multirow[t]{2}{*}{ AA UMa } & 0.468 & 0.547 & $\mathrm{~A}$ & G0 & 1.26 & 1.40 & 5932 & 2.17 & 3,6 & \\
\hline & 11.30 & 3.168 & B & & 1.10 & 0.69 & 6030 & 1.43 & & \\
\hline AW UMa & 0.439 & 0.349 & $\mathrm{~A}$ & & 1.52 & 1.60 & 7175 & 6.06 & $1,2,18$ & \\
\hline HD 99946 & 7.27 & 3.221 & B & & 0.53 & 0.11 & 6875 & 0.56 & & \\
\hline RZ Pyx & 0.656 & 0.821 & $\mathrm{~A}$ & B7V & 5.76 & 2.69 & 4.230 & 2.73 & 21 & \\
\hline HD 75920 & 8.85 & 6.954 & B & & 4.73 & 2.51 & 4.225 & 2.65 & & \\
\hline
\end{tabular}

References: 1: Fracastoro (1972), 2: Mochnacki (1981), 3: Maceroni \& Van 'T Veer (1996), 4: Hrivnak (1989), 5: Leung \& Schneider (1978), 6: Barone et al. (1993), 7: Maceroni (1986), 8: Samec (1987), 9: Lapasset \& Claria (1986), 10: Bradstreet (1985), 11: Samec et al. (1995), 12: Maceroni et al. (1994), 13: Lapasset \& Gomez (1990), 14: Cerruti-Sola et al. (1981), 15: Zhai et al. (1988), 16: Samec \& Terrell (1995), 17: Wilson \& Devinney (1973), 18: Morris \& Naftilan (1997), 19: Lapasset et al. (1996), 20: Bell \& Malcolm (1987). 